Algebraic 85 Geometric Topology

Volume 2 (2002) 949-1000

Published: 25 October 2002

ATG

\title{
Configuration spaces and Vassiliev classes in any dimension
}

\author{
Alberto S. Cattaneo \\ PaOlo Cotta-Ramusino \\ RiCCARDO LONGONI
}

\begin{abstract}
The real cohomology of the space of imbeddings of $S^{1}$ into $\mathbb{R}^{n}$, $n>3$, is studied by using configuration space integrals. Nontrivial classes are explicitly constructed. As a by-product, we prove the nontriviality of certain cycles of imbeddings obtained by blowing up transversal double points in immersions. These cohomology classes generalize in a nontrivial way the Vassiliev knot invariants. Other nontrivial classes are constructed by considering the restriction of classes defined on the corresponding spaces of immersions.
\end{abstract}

AMS Classification 58D10; 55R80, 81Q30

Keywords Configuration spaces, Vassiliev invariants, de Rham cohomology of spaces of imbeddings and immersions, Chen's iterated integrals, graph cohomology

\section{Introduction}

In this paper we study de Rham cohomology classes of the space $\operatorname{Imb}\left(S^{1}, \mathbb{R}^{n}\right)$ of smooth imbeddings of $S^{1}$ into $\mathbb{R}^{n}, n>3$, using as main tools configurationspace integrals and graph cohomology.

Before describing the setting of this paper we give a brief description of the main results obtained.

\section{$1.1 \quad$ Main results}

We consider two complexes $\left(\mathcal{D}_{o}^{k, m}, \delta_{o}\right)$ and $\left(\mathcal{D}_{e}^{k, m}, \delta_{e}\right)$ generated by some decorated graphs. These graph complexes are bigraded by two integers $m, k$ called respectively the degree and the order. The order is minus the Euler characteristic of the graph, while the degree measures the deviation of the graph from 
being trivalent. The coboundary operators increase the degree by one and do not change the order.

We prove in subsection 6.1 the following:

Theorem 1.1 For every $k \in \mathbb{N}$, there exist chain maps from graph complexes to de Rham complexes

$$
\begin{array}{ll}
\mathcal{D}_{o}^{k, m} \rightarrow \Omega^{(n-3) k+m}\left(\operatorname{Imb}\left(S^{1}, \mathbb{R}^{n}\right)\right) & \text { for } n \text { odd } \\
\mathcal{D}_{e}^{k, m} \rightarrow \Omega^{(n-3) k+m}\left(\operatorname{Imb}\left(S^{1}, \mathbb{R}^{n}\right)\right) & \text { for } n \text { even }
\end{array}
$$

that induce injective maps in cohomology when $m=0$.

From the combinatorial structure of the graph complexes, one immediately deduces (see again subsection 6.1) the following:

Corollary 1.2 For any $n>3$ and for any positive integer $k_{0}$, there are nontrivial cohomology classes on $\operatorname{Imb}\left(S^{1}, \mathbb{R}^{n}\right)$ of degree greater than $k_{0}$.

All the differential forms appearing in the above Theorem turn out to be equivariant w.r.t. the action of the group $\operatorname{Diff}^{+}\left(S^{1}\right)$ of orientation preserving diffeomorphisms of the circle.

The classes of Theorem 1.1 can be seen as an extension to higher dimensions of Vassiliev knot invariants. One of the main ingredients of Vassiliev's approach is to consider immersions which are imbeddings but for a finite number (say $k$ ) of transversal double points (let us call them "special immersions"). The ways of pushing off a double point form, up to homotopy, an $(n-3)$-dimensional sphere (viz., one must choose a normalized vector transverse to the plane spanned by the tangent vectors of the intersecting strands at the double point). So every special immersion with $k$ double points gives rise to a $k(n-3)$-cycle in $\operatorname{Imb}\left(S^{1}, \mathbb{R}^{n}\right)$. Our construction allows us to prove that infinitely many of these cycles are nontrivial.

When we extend the above construction to imbeddings with framing, namely when we consider pairs $(K, w)$ consisting of an imbedding $K: S^{1} \rightarrow \mathbb{R}^{n}$ and a section $w$ of the pulled-back bundle $K^{*} S O\left(\mathbb{R}^{n}\right)$, then the situation becomes simpler and we prove in subsection 7.4 the following:

Theorem 1.3 All cycles of framed imbeddings of $S^{1}$ into $\mathbb{R}^{2 s+1}$ determined by framed special immersions are nontrivial. 
The restriction of cohomology classes on the space of immersions $\operatorname{Imm}\left(S^{1}, \mathbb{R}^{n}\right)$ to the space of imbeddings $\operatorname{Imb}\left(S^{1}, \mathbb{R}^{n}\right)$ is also discussed. Combining Theorems 8.5, Proposition 8.6 and Corollary 8.7 we obtain:

Theorem 1.4 When $n$ is odd, the inclusion $\operatorname{Imb}\left(S^{1}, \mathbb{R}^{n}\right) \hookrightarrow \operatorname{Imm}\left(S^{1}, \mathbb{R}^{n}\right)$ induces the zero map in cohomology. When $n$ is even, the inclusion map $\operatorname{Imb}\left(S^{1}, \mathbb{R}^{n}\right) \hookrightarrow \operatorname{Imm}\left(S^{1}, \mathbb{R}^{n}\right)$ is nontrivial in cohomology.

Contrary to what happens in Thm. 1.1, not all the differential forms of Thm. 1.4 are $\operatorname{Diff}^{+}\left(S^{1}\right)$-equivariant. However, the equivariant ones turn out to be in the image of certain graphs (of degree different from zero) through the map (1.2). Thus, Thm. 1.4 provides an extension of the last statement of Thm. 1.1 in the case $m \neq 0$.

\section{$1.2 \quad$ The setting}

The configuration spaces $C_{q}^{0}(M)$ of a manifold $M$ are simply the Cartesian products $M^{q}$ minus all diagonals. Configuration spaces are naturally associated to imbeddings. Indeed, if $f: N \rightarrow M$ is an imbedding, it defines maps $C_{q}^{0}(f): C_{q}^{0}(N) \rightarrow C_{q}^{0}(M)$ for every $q \geq 0$.

This simple, natural relation has an application to knot invariants, i.e., to the study of the zeroth cohomology of the space of imbeddings of $S^{1}$ into $\mathbb{R}^{3}$. We refer to Bott and Taubes's [9] construction inspired from the perturbative expansion of Chern-Simons theory [37]. The "physical" origin of this construction should not be too surprising; for, as a matter of fact, a "correlation function" in physics (i.e., the inverse of some differential operator) is usually well-defined only at non-coincident points - and this also leads naturally to configuration spaces.

The gist of their construction is as follows: One considers differential forms on $C_{q}^{0}\left(\mathbb{R}^{3}\right)$ given by products of the rotational-invariant representatives of the two-dimensional generators in cohomology ("tautological forms"); next one integrates these forms on cycles defined by constraining some of the $q$ points to lie in the image of the given imbedding $K$; finally, one wants to prove that certain linear combinations of these integrals are actually invariant under isotopies of $K$.

The big technical problem in Bott and Taubes's construction (as well as in other related constructions $[3,26,7,8]$ for 3 -manifold invariants) is the convergence of the above integrals, a nontrivial fact since the tautological forms 
are not compactly supported. An elegant solution-which also lies at the core of the subsequent construction of invariants by determining the suitable linear combinations of integrals - relies on a compactification of configuration spaces on which the tautological forms extend as smooth forms. This is the FultonMacPherson [20] compactification, but in the differential-geometric version later given by Axelrod and Singer [3]. What is actually needed is still a further improvement; viz., a compactification of the configuration spaces of $\mathbb{R}^{3}$ with some points lying on the knot. This is done in [9], and indeed in the more general case of the configuration space of a manifold $M$ with some points lying on the image of a given imbedding of another manifold $N$ (assuming $N$ and $M$ to be compact). The last result allows one to approach more general imbedding problems, as is done in the present work. We finally observe that, in the 3 dimensional case, only knot invariants have been constructed this way (and no higher-degree cohomology classes on the space of imbeddings) and, moreover, that these invariants are proved to exist and to be nontrivial, but are obtained modulo corrections with unknown coefficients.

Let us now turn to the case of imbeddings of $S^{1}$ into $\mathbb{R}^{n}$ with $n>3$. Here "tautological forms" are representatives of the $(n-1)$-dimensional cohomology generators of configuration spaces of $\mathbb{R}^{n}$. We integrate products of tautological forms on cycles in configuration spaces constraining some of the points to lie on the imbedding, thus getting differential forms on $\operatorname{Imb}\left(S^{1}, \mathbb{R}^{n}\right)$. We construct closed linear combinations of these forms by using graph cohomology as explained in the following.

Graphs, with edges corresponding to tautological forms, are a simple way of keeping track of all configuration-space integrals one may consider. In order to get a complete description, i.e. without sign ambiguities, one actually has to decorate the graphs in a certain way (in fact, two different ways corresponding to $n$ even or odd). At this point, one can define a grading and a coboundary operator on the vector space generated by all decorated graphs in such a way that the assignment to each graph of the corresponding configuration-space integral defines a (degree shifting) chain map from the graph complex to de Rham complex of $\operatorname{Imb}\left(S^{1}, \mathbb{R}^{n}\right)$, see Theorem 4.4. We give an explicit definition of graph cohomologies, along the lines originally proposed by Kontsevich [26], and describe in details the chain maps and so the relation to the problem of imbeddings. It is at this point that it is crucial to have $n>3$, as for $n=3$ the map we construct might fail to be a chain map. As was observed by the referee, this chain map is also a morphism of differential graded commutative algebras, with the multiplication on the graphs defined as (a graded version of) the shuffle product. We plan to return on this and to discuss other algebraic structures 
on the graph complex in [12]. Finally, we prove that the induced map in cohomology is injective in degree zero. This is done by pairing the corresponding cohomology classes on $\operatorname{Imb}\left(S^{1}, \mathbb{R}^{n}\right)$ to the cycles arising from special immersions described before. Observe that, for $n>3$, the connected components of the space of special immersions are in one-to-one correspondence with chord diagrams (i.e., graphs consisting of a distinguished circle with chords), each chord representing a transversal double point. We prove that the pairing of a cycle of imbeddings determined by a special immersion with a differential form coming from a graph cocycle containing the corresponding chord diagram is non zero, see Theorem 6.4. Moreover, we prove that every graph cocycle of degree zero contains a chord diagram, see Proposition 5.1.

Consider now the space $\operatorname{Imb}_{\mathrm{f}}\left(S^{1}, \mathbb{R}^{n}\right)$ of framed imbeddings. Since we have a projection to $\operatorname{Imb}\left(S^{1}, \mathbb{R}^{n}\right)$ (forgetting the framing), we can pull back all cohomology classes constructed before. Given a framed special immersion, we can then generate a cycle of framed imbeddings and exactly as above prove that cohomology classes corresponding to graph cocycles in degree zero are nontrivial. In odd dimensions, we can extend our construction and produce new classes (corresponding to new classes in a modified graph cohomology), and again prove nontriviality in degree zero. But at this point, we can also use the same technique to prove that all cycles determined by framed special immersions are nontrivial, as stated in the Theorem at the beginning.

A problem which is strictly related to the subject of this paper is the study of the cohomology of the spaces of long knots, i.e., the spaces of imbeddings of the real line into Euclidean spaces with fixed behavior at infinity. This problem has been already addressed by various authors $[22,30,33,36]$. We comment here that the methods developed in this paper are easily generalized in that direction. In particular one can prove [34] that our graph complexes are quasi-isomorphic to the first term of the spectral sequences defined by Vassiliev $[33,36]$ and Sinha [30], and that Theorem 1.1 implies the convergence of these spectral sequences along the diagonal.

We conclude with some remarks on the relation between the configuration-space techniques described above and physics. We recall that in the 3-dimensional case, Vassiliev knot invariants appear in the perturbative expansion of expectation values of traces of holonomies in Chern-Simons theory [37]. Bott and Taubes's construction is based on the expansion in the "covariant gauge" [23, 4], whereas the Kontsevich integral $[25,5]$ is based on the expansion in the "holomorphic gauge" [19]. As described in [11], the same Vassiliev invariants may also be obtained in the perturbative expansion of $B F$ theory in 3 dimensions. 
This theory can actually be defined in any dimension, and the perturbative expansion of expectation values of traces of the generalized holonomies defined in $[14,16]$ (see also [13]) are actually related to the cohomology classes of imbeddings described in the present paper. Moreover, the analysis of the $B F$ theories made in [15] suggests the possibility of connecting our results with the string topology of Chas and Sullivan [17].

\subsection{Plan of the paper}

In Section 2 we define a map that assigns to each element of $H^{p}\left(\operatorname{Imb}\left(S^{1}, \mathbb{R}^{n}\right), \mathbb{R}\right)$ a cohomology class in $H^{p-k(n-3)}\left(\operatorname{Imm}_{k}\left(S^{1}, \mathbb{R}^{n}\right), \mathbb{R}\right)$ where $\operatorname{Imm}_{k}\left(S^{1}, \mathbb{R}^{n}\right)$ denotes the space of immersion with exactly $k$ transversal double points. This map is the generalization of the map that extends knot invariants to invariants of $\operatorname{Imm}_{k}\left(S^{1}, \mathbb{R}^{3}\right)[35,5]$. We say then that a real cohomology class of $\operatorname{Imb}\left(S^{1}, \mathbb{R}^{n}\right)$ has Vassiliev-order $s$ if the corresponding cohomology class of $\operatorname{Imm}_{k}\left(S^{1}, \mathbb{R}^{n}\right)$ is zero for $k>s$ and non-zero for $k=s$.

After recalling the Bott-Taubes construction for tautological forms and configuration spaces in Section 3, we define in Section 4 the two complexes $\left(\mathcal{D}_{o}, \delta_{o}\right)$ and $\left(\mathcal{D}_{e}, \delta_{e}\right)$ mentioned above. We show that the configuration space integral is a chain map from the above complexes to the de Rham complex of $\operatorname{Imb}\left(S^{1}, \mathbb{R}^{n}\right)$ (where the two cases $n$ even and $n$ odd are kept separately).

In Section 5 we focus on trivalent graphs and construct explicitly some nontrivial cocycles that are given by linear combinations of them.

In Section 6 we show that for $n>3$ the morphisms between the complexes $\left(\mathcal{D}_{o}, \delta_{o}\right),\left(\mathcal{D}_{e}, \delta_{e}\right)$ and $\left(\Omega^{*}\left(\operatorname{Imb}\left(S^{1}, \mathbb{R}^{n}\right)\right), d\right)$ ( $n$ odd and, respectively, even) are monomorphisms in cohomology when they are restricted to the subspaces of trivalent graphs. At the end, we prove Thm. 1.1 and Cor. 1.2.

In Section 7 we consider the space of framed imbeddings $\operatorname{Imb}_{\mathrm{f}}\left(S^{1}, \mathbb{R}^{n}\right)$. We show how to define new classes in case $n$ is odd. Here we define a modified graph cohomology and a new chain map, which we prove to be injective in cohomology in degree zero. We also prove Thm. 1.3.

In Section 8 we recall the construction of the generators of $H^{*}\left(\operatorname{Imm}\left(S^{1}, \mathbb{R}^{n}\right), \mathbb{R}\right)$ via Chen integrals [18] and compute their restrictions to $\operatorname{Imb}\left(S^{1}, \mathbb{R}^{n}\right)$. We show that this restriction is trivial if $n$ is odd but yields nontrivial classes of $\operatorname{Imb}\left(S^{1}, \mathbb{R}^{n}\right)$ if $n$ even, thus proving Thm. 1.4.

Finally, in the Appendix we discuss some Vanishing Theorems that are needed in order to define the morphisms between the complexes considered before. The 
main result is that, in computing the differential of an integral of tautological forms, contributions from the so-called hidden faces are always zero.

Conventions Throughout this paper we assume $n>3$, unless otherwise stated.

We also assume that all the spaces under consideration (namely, $S^{1}$ and $\mathbb{R}^{n}$ ) are oriented. In particular two imbeddings (or immersions) that are obtained from each other by reversing the orientation of $S^{1}$ will be considered as different elements of $\operatorname{Imb}\left(S^{1}, \mathbb{R}^{n}\right)$ (or $\left.\operatorname{Imm}\left(S^{1}, \mathbb{R}^{n}\right)\right)$.

We are concerned only with real cohomology groups that we will denote by $H^{*}\left(\operatorname{Imb}\left(S^{1}, \mathbb{R}^{n}\right)\right)$ or $H^{*}\left(\operatorname{Imm}\left(S^{1}, \mathbb{R}^{n}\right)\right)$.

Finally, in the course of the paper we need to choose a unit generator of the top cohomology of $S^{n}$. The main results of Section 8 are independent of such choice. In the rest of this paper, however, we need to restrict ourselves to symmetric forms (see Definition 4.3).

Acknowledgments We thank especially Raoul Bott, Jim Stasheff, Victor Vassiliev and the referee for pointing out parts of the previous version of this paper that needed clarification or corrections. We thank Giovanni Felder, Nathan Habegger, Maurizio Rinaldi, Dev Sinha, Dennis Sullivan and Victor Tourtchine for useful comments and interesting discussions. We thank Carlo Rossi and Simone Mosconi for carefully reading the manuscript.

A. S. C. thanks the I.N.F.N., Sezione di Milano, P. C.-R. thanks the Universität Zürich-Irchel and the ETH Zürich, and R. L. thanks the Universität ZürichIrchel for their hospitality.

A. S. C. thanks partial support of SNF Grant No. $\backslash 2100-055536.98 / 1$. P. C.-R. and R. L. thank partial support of MURST.

\section{Vassiliev classes in $H^{*}\left(\operatorname{Imb}\left(S^{1}, \mathbb{R}^{n}\right)\right)$}

In this Section we propose a classification scheme for the cohomology classes in $H^{*}\left(\operatorname{Imb}\left(S^{1}, \mathbb{R}^{n}\right)\right)$, including those that not are necessarily obtained by pullback of classes in $H^{*}\left(\operatorname{Imm}\left(S^{1}, \mathbb{R}^{n}\right)\right)$ via the inclusion map

$$
\operatorname{Imb}\left(S^{1}, \mathbb{R}^{n}\right) \hookrightarrow \operatorname{Imm}\left(S^{1}, \mathbb{R}^{n}\right) .
$$

This scheme is a direct generalization of the scheme proposed by Vassiliev [35] for knot invariants in $\mathbb{R}^{3}$. 
We consider the space $\operatorname{Imm}_{k}\left(S^{1}, \mathbb{R}^{n}\right)$ which is defined as the submanifold of $\operatorname{Imm}\left(S^{1}, \mathbb{R}^{n}\right)$ whose elements have exactly $k$ transversal double points. Moreover we set $\operatorname{Imm}_{k}^{\prime}\left(S^{1}, \mathbb{R}^{n}\right)$ to be the submanifold of $\operatorname{Imm}_{k}\left(S^{1}, \mathbb{R}^{n}\right)$ given by those immersions whose initial point $\gamma(0)$ does not coincide with any double point.

We enumerate all the double points of any $\gamma \in \operatorname{Imm}_{k}^{\prime}\left(S^{1}, \mathbb{R}^{n}\right)$ starting from the initial point $\gamma(0)$. Then we blow up, in order, all the double points in the way described below.

Let $\mathbf{x}^{j}=\gamma\left(t_{1}^{j}\right)=\gamma\left(t_{2}^{j}\right)$ be the $j$ th double point, with $t_{1}^{j}<t_{2}^{j}$. We denote by $l_{1}^{j}=D \gamma\left(t_{1}^{j}\right)$ and $l_{2}^{j}=D \gamma\left(t_{2}^{j}\right)$ the normalized tangent vectors at $\mathbf{x}^{j}$ and by $T^{j}$ the plane in $T_{\mathbf{x}^{j}} \mathbb{R}^{n}$ spanned by $l_{1}^{j}$ and $l_{2}^{j}$ with the orientation determined by $l_{1}^{j} \wedge l_{2}^{j}$.

Here we assume to have chosen once and for all an orientation in $\mathbb{R}^{n}$. Moreover, for the rest of this section it is useful to pick up a metric on $\mathbb{R}^{n}$ as well.

Then we consider the $(n-2)$-plane $N^{j} \subset T_{\mathbf{x}^{j}} \mathbb{R}^{n}$ that is normal to $T^{j}$ with the induced orientation and the space $Q^{j}$ of normalized vectors in $N^{j}$.

The space $\mathfrak{Q}_{k}^{j}$ of pairs $\left(\gamma, \mathbf{z}^{\mathbf{j}}\right)$ of immersions with $k$ transversal double points and normalized vectors in $Q^{j}$ can be formally described as follows. If we consider the Grassmann manifold $S G_{2, n}$ of oriented 2-planes in $\mathbb{R}^{n}$, then we have smooth maps

$$
r_{k, j}: \operatorname{Imm}_{k}^{\prime}\left(S^{1}, \mathbb{R}^{n}\right) \rightarrow S G_{2, n} \equiv S O(n) /\{S O(2) \times S O(n-2)\}
$$

that associate to the $j$-th double point the oriented plane $T^{j}$.

We have an associated bundle

$$
Q=S O(n) \times\{S O(2) \times S O(n-2)\} S^{n-3} \rightarrow S G_{2, n}
$$

whose fiber is the homogeneous space

$$
S^{n-3}=[S O(2) \times S O(n-2)] /[S O(2) \times S O(n-3)] \equiv S O(n-2) / S O(n-3) .
$$

The space $Q$ can equivalently be obtained by dividing $S O(n)$ by $S O(2) \times$ $S O(n-3)$.

The pull-back bundle $\mathfrak{Q}_{k}^{j} \equiv r_{k, j}^{*} Q$ is a sphere bundle with fiber $S^{n-3}$ so that the following diagram:

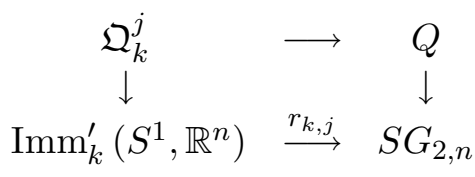

Algebraic 83 Geometric Topology, Volume 2 (2002) 
is commutative.

By considering in their order all the double points, we can define the map

$$
r_{k}: \operatorname{Imm}_{k}^{\prime}\left(S^{1}, \mathbb{R}^{n}\right) \rightarrow\left(S G_{2, n}\right)^{\times k}
$$

and the bundle

$$
\mathfrak{Q}_{k} \equiv r_{k}^{*} Q^{\times k}
$$

with fiber $\left(S^{n-3}\right)^{\times k}$

Next we will define, see (2.3), a map $s_{k}: \mathfrak{Q}_{k} \rightarrow \operatorname{Imb}\left(S^{1}, \mathbb{R}^{n}\right)$ that corresponds to the blow-up of all the double points of an immersion.

Given $\gamma \in \operatorname{Imm}_{k}^{\prime}\left(S^{1}, \mathbb{R}^{n}\right)$ and an element of the fiber of $\mathfrak{Q}_{k}^{i}$ over $\gamma$, which we represent as $\mathbf{z}^{j} \in S^{n-3}$, we choose $a_{j}$ to be either 1 or 2 and define the following loops in $T_{\mathbf{x}^{j}} \mathbb{R}^{n}$ :

$$
\alpha_{a_{j}}^{j}\left(\mathbf{z}^{j}\right)(t)= \begin{cases}0 & \text { if } t \notin\left[t_{a_{j}}^{j}-\epsilon, t_{a_{j}}^{j}+\epsilon\right], \\ (-1)^{a_{j}+1} \mathbf{z}^{j} \delta \exp \left(1 /\left[\left(t-t_{a_{j}}^{j}\right)^{2}-\epsilon^{2}\right]\right) & \text { if } t \in\left[t_{a_{j}}^{j}-\epsilon, t_{a_{j}}^{j}+\epsilon\right],\end{cases}
$$

with $\epsilon, \delta>0$.

If we add to the immersion $\gamma$ the loop $\alpha_{a_{j}}^{j}\left(\mathbf{z}^{j}\right)$, using the natural identification $\mathbb{R}^{n} \approx T_{\mathbf{x}^{j}} \mathbb{R}^{n}$, we remove the $j$ th double point (see figure 1 ).

We assume, from now on, that the parameters $\epsilon$ and $\delta$ are chosen so small that no new double point is created by this operation.

In this construction one of the two strands that meet in the $j$ th double point is "lifted" in a way parameterized by $\mathbf{z}^{j}$ that belongs to the fiber over $\gamma$ of the sphere bundle $\mathfrak{Q}_{k}^{j}$. The union of all the possible lifts (for a given immersion $\gamma$ and a given double point) describes the suspension of the fiber $S^{n-3}$, namely, an $(n-2)$-sphere $\mathcal{S}_{a_{j}}^{j}$. Denoting by $\ell_{a_{j}}^{j}$ the straight line passing through $\mathbf{x}^{j}$ with tangent $l_{a_{j}}^{j}$, we have the following

Proposition 2.1 The linking number between $\ell_{a_{j}}^{j}$ and $\mathcal{S}_{b_{j}}^{j}, b_{j} \equiv a_{j}+1$ $\bmod 2$, is one.

The proof is just a consequence of the orientation choices. Observe that, being $\ell_{a_{j}}^{j}$ a 1-manifold, the above linking number does not depend on the order.

For any given choice of $a$ and of the "small" parameters $\epsilon$ and $\delta$ at each double point, we have thus defined a map

$$
s_{k}: \mathfrak{Q}_{k} \rightarrow \operatorname{Imb}\left(S^{1}, \mathbb{R}^{n}\right)
$$


which is described, in any coordinate neighborhood of $\gamma \in \operatorname{Imm}_{k}^{\prime}\left(S^{1}, \mathbb{R}^{n}\right)$, by cycles:

$$
\left(S^{n-3}\right)^{k} \ni\left(\mathbf{z}^{1}, \cdots, \mathbf{z}^{k}\right) \mapsto \gamma+\sum_{j=1}^{k} \alpha_{a_{j}}^{j}\left(\mathbf{z}^{j}\right) .
$$

Due to the arbitrariness in the choice of the index $a_{j} \in\{1,2\}$ attached to each double point of $\gamma \in \operatorname{Imm}_{k}^{\prime}\left(S^{1}, \mathbb{R}^{n}\right)$, we have constructed $2^{k}$ cycles, for which we have the following

Proposition 2.2 If we choose different values of $a_{j} \in\{1,2\}$ for the double point labelled by $j$ in (2.2), then the resulting cycles (2.4) are homologous.

Proof It is enough to consider two segments $[0,1] \ni t \mapsto l_{1}^{j}(t)$ and $[0,1] \ni s \mapsto$ $l_{2}^{j}(s)$ that intersect transversally at the middle point. We choose $\mathbf{z}^{j} \in S^{n-3}$ and remove the crossing point as follows:

$$
\left\{\begin{array}{l}
l_{1}^{j}(t) \mapsto l_{1}^{j}(t)+\mathbf{z}^{j} \delta \exp \left(1 /\left[(t-1 / 2)^{2}-\epsilon^{2}\right]\right) \\
l_{2}^{j}(s) \mapsto l_{2}^{j}(s)-\mathbf{z}^{j} \delta \exp \left(1 /\left[(s-1 / 2)^{2}-\epsilon^{2}\right]\right)
\end{array}\right.
$$

where $\delta$ and $\epsilon$ are small positive numbers.
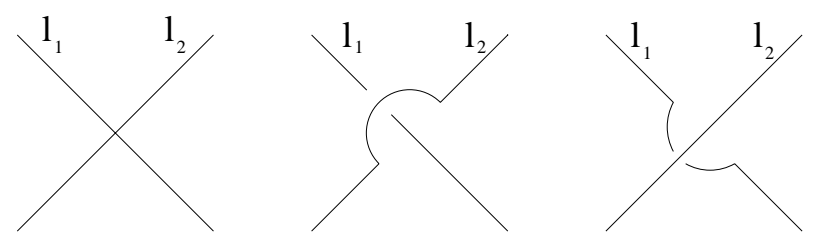

Figure 1: The resolution of a transversal double intersection

We have then an $(n-3)$-cycle of imbedded pairs of segments with fixed endpoints. Let us now take $h \in[0,1]$. If we replace $\delta$ with $h \delta$ in the first line of (2.5), then we have a homotopy between the cycle (2.5) and the cycle obtained by modifying only $l_{2}^{j}$. Analogously if we replace $\delta$ with $h \delta$ in the second line of (2.5), we have an homotopy between (2.5) and the cycle obtained by modifying only $l_{1}^{j}$.

By pulling back cohomology classes via (2.3) and integrating them along the fibers in $\mathfrak{Q}_{k}$ we obtain the following morphisms in cohomology:

$$
i_{k}^{\prime}: H^{p}\left(\operatorname{Imb}\left(S^{1}, \mathbb{R}^{n}\right)\right) \rightarrow H^{p-k(n-3)}\left(\operatorname{Imm}_{k}^{\prime}\left(S^{1}, \mathbb{R}^{n}\right)\right) .
$$


Notice that the maps (2.6) are independent of the choices of the $a$ 's at each double point.

For future purposes, we extend the map (2.6) by setting it equal to zero if $p-k(n-3)<0$. Hence $i_{k}^{\prime}$ is defined for every $k \in \mathbb{N}$.

Definition 2.3 We say that a cohomology class $\omega \in H^{p}\left(\operatorname{Imb}\left(S^{1}, \mathbb{R}^{n}\right)\right)$ is of finite Vassiliev-order (or V-order) $k$ if $i_{s}^{\prime}(\omega)=0$ for every $s>k$ and $i_{k}^{\prime}(\omega) \neq 0$. If $i_{k}^{\prime}(\omega)$ is non zero for any $k$, then we say that the V-order is infinite.

Remark 2.4 If $n>3$, then the $\mathrm{V}$-order is always finite. If $n=3$ then the $\mathrm{V}$ order may be infinite. The case $n=3$ and $p=0$ is the case of knot invariants, as originally studied by Vassiliev [35].

If $n>3$ and $p=k(n-3)$, then from (2.6) we conclude that there is a morphism:

$$
i_{k}^{\prime}: H^{k(n-3)}\left(\operatorname{Imb}\left(S^{1}, \mathbb{R}^{n}\right)\right) \rightarrow H^{0}\left(\operatorname{Imm}_{k}^{\prime}\left(S^{1}, \mathbb{R}^{n}\right)\right) .
$$

This case will be particularly important in the rest of the paper, basically because of the following

Proposition 2.5 If $n>3$, then:

(i) the connected components of $\operatorname{Imm}_{k}\left(S^{1}, \mathbb{R}^{n}\right)$ are in one-to-one correspondence with the set of chord diagrams with $k$ chords;

(ii) the connected components of $\operatorname{Imm}_{k}^{\prime}\left(S^{1}, \mathbb{R}^{n}\right)$ are in one-to-one correspondence with chord diagrams with $k$ chords and a marked point distinct from the end-points of the chords.

Here and in the following, by chord diagram we mean a circle with chords that have no end-points in common.

Proof If $n>3$, then any finite collection of (piecewise) imbedded loops can be isotopically deformed to a trivial link. Hence the connected components of $\operatorname{Imm}_{k}\left(S^{1}, \mathbb{R}^{n}\right)$ are determined uniquely by the position of the double points. Their pre-images are points on a circle that are identified in pairs, i.e., chords.

In the case of $\operatorname{Imm}_{k}^{\prime}\left(S^{1}, \mathbb{R}^{n}\right)$, one has just to take care of the additional information given by the initial point.

In general we want to determine whether a given class in $H^{p}\left(\operatorname{Imb}\left(S^{1}, \mathbb{R}^{n}\right)\right)$ is trivial or not. The relevance of the order $p=k(n-3)$ is highlighted by the following criterion: 
Corollary 2.6 A sufficient condition for a class $\omega \in H^{k(n-3)}\left(\operatorname{Imb}\left(S^{1}, \mathbb{R}^{n}\right)\right)$ to be nontrivial is that its image under (2.7) is nontrivial.

Remark 2.7 We have a map

$$
\varphi: H_{0}\left(\operatorname{Imm}_{k}\left(S^{1}, \mathbb{R}^{n}\right)\right) \rightarrow H_{0}\left(\operatorname{Imm}_{k}^{\prime}\left(S^{1}, \mathbb{R}^{n}\right)\right)
$$

which associates to any chord diagram $D$ the average of all inequivalent chord diagrams with a marked point that have the same chords of $D$. This map has a right inverse, viz., the map

$$
F: H_{0}\left(\operatorname{Imm}_{k}^{\prime}\left(S^{1}, \mathbb{R}^{n}\right)\right) \rightarrow H_{0}\left(\operatorname{Imm}_{k}\left(S^{1}, \mathbb{R}^{n}\right)\right)
$$

that forgets the marked point.

In the following we will consider the combination of $(2.7)$ with $\varphi^{*}$ thus obtaining a map:

$$
i_{k}: H^{k(n-3)}\left(\operatorname{Imb}\left(S^{1}, \mathbb{R}^{n}\right)\right) \rightarrow H^{0}\left(\operatorname{Imm}_{k}\left(S^{1}, \mathbb{R}^{n}\right)\right) .
$$

A class $\omega$ in $H^{0}\left(\operatorname{Imm}_{k}^{\prime}\left(S^{1}, \mathbb{R}^{n}\right)\right)$ will be called equivariant if $F^{*} \varphi^{*} \omega=\omega$.

Classes in $H^{k(n-3)}\left(\operatorname{Imb}\left(S^{1}, \mathbb{R}^{n}\right)\right)$ can be constructed via trivalent graphs, as shown in the sequel of this paper. These classes have been firstly considered in the 3-dimensional case, in connection with perturbative Chern-Simons quantum field theory.

\section{The Bott-Taubes construction}

\subsection{Configuration spaces}

For any compact manifold $M$, we consider first the configuration space $C_{q}^{0}(M)$ $\triangleq M^{q} \backslash\left\{\bigcup_{S} \Delta_{S}\right\}$, where $S$ runs over the ordered subsets of the first $q$ integers with $|S| \geq 2$, and $\Delta_{S}$ denotes the (multi)-diagonal labelled by $S$, namely, the subset of $M^{q}$ defined by the equations $x_{j_{1}}=x_{j_{2}}=\cdots=x_{j_{|S|}}, j_{i} \in S$.

We consider then the compactification $C_{q}(M)$ of $C_{q}^{0}(M)$ introduced in [3] as a modification of the Fulton-MacPherson construction [20], as described below.

One has an obvious inclusion of $C_{q}^{0}(M) \subset M^{q}$ and, for each diagonal $\Delta_{S}$, one has a projection $C_{q}^{0}(M) \rightarrow B l\left(M^{|S|}, \Delta_{S}\right)$ where $B l$ denotes the differentialgeometric blowup (i.e., one replaces the given diagonal $\Delta_{S}$ by the sphere bundle of its normal bundle). This gives an imbedding $C_{q}^{0}(M) \hookrightarrow M^{q} \times$ $\prod_{|S| \geq 2} B l\left(M^{|S|}, \Delta_{S}\right)$. The space $C^{q}(M)$ is then defined as the closure of $C_{q}^{0}(M)$ in the above space. The main fact about this compactification of configuration spaces (see [9]) is the following: 
Theorem 3.1 The spaces $C_{q}(M)$ are smooth manifolds with corners, and all the projections $C_{q}^{0}(M) \rightarrow C_{q-k}^{0}(M)$ extend to smooth projections on the corresponding compactified spaces.

The boundaries of $C_{q}(M)$ correspond to the "collision" of at least two of the $q$ points of $M$. Boundaries are the union of different strata corresponding to the different ways in which all the points may collide. More precisely, let $S \subset\{1, \cdots, q\}$ be the labels of the colliding points. Let us insert in $S$ different levels of parentheses so that each pair of parentheses contains at least two elements. Points in $M$ "collide at the same speed" if they belong to the same level of parentheses (points are assumed to "collide" starting from the innermost parentheses). The codimension of a given stratum is equal to the number of pairs of parentheses.

We are mainly interested in codimension-1 strata, namely, in those strata with no internal parentheses. For these strata, one calls hidden faces those corresponding to subsets $S$ with $|S| \geq 3$ and principal faces those for which $|S|=2$.

\subsubsection{The case of $S^{1}$}

If we choose $M$ to be $S^{1}$, then $C_{q}^{0}\left(S^{1}\right)$ is not connected. We choose a connected component by fixing an order of the points on $S^{1}$ (consistent with its orientation). It is then easy to see that this connected component is given by $S^{1} \times \Sigma_{q-1}^{0}$ where $\Sigma_{q-1}^{0}$ is the ordinary open $(q-1)$-dimensional simplex. We denote the closure of the connected component of $C_{q}^{0}\left(S^{1}\right)$ by the symbol $C_{q}$. This is given by the Cartesian product of $S^{1}$ times a space $W_{q-1}$ that is obtained from the standard closed $(q-1)$-simplex by a sequence of blowups (see the explicit description in [9]).

\subsubsection{The case of $\mathbb{R}^{n}$}

In the following we need a suitable compactification of $C_{q}^{0}\left(\mathbb{R}^{n}\right)$. Since $\mathbb{R}^{n}$ is not compact, we cannot rely directly on the preceding construction.

Instead, following [9], we identify $\mathbb{R}^{n}$ with $S^{n} \backslash\{\infty\}$ and define $C_{q}\left(\mathbb{R}^{n}\right)$ as the fiber over $\infty \in S^{n}$ of $C_{q+1}\left(S^{n}\right) \rightarrow S^{n}$ (say, projecting to the last factor).

This way, we also have a compactification (and corresponding boundary faces) at infinity. (For example, $C_{1}\left(\mathbb{R}^{n}\right)$ is the $n$-dimensional ball.) 


\subsubsection{The case of an imbedding of $S^{1}$ into $\mathbb{R}^{n}$}

This is the case of interest for the rest of the paper.

Again following [9], we define the space $C_{q, t}\left(\mathbb{R}^{n}\right)$ of $q+t$ distinct points in $\mathbb{R}^{n}$, the first $q$ of which are constrained on an imbedding of $S^{1}$, as a pulled-back bundle as follows:

$$
\begin{array}{ccc}
C_{q, t}\left(\mathbb{R}^{n}\right) & \stackrel{\hat{e v}}{\longrightarrow} & C_{q+t}\left(\mathbb{R}^{n}\right) \\
\downarrow p_{1} & & \downarrow \\
C_{q} \times \operatorname{Imb}\left(S^{1}, \mathbb{R}^{n}\right) & \stackrel{e v}{\longrightarrow} & C_{q}\left(\mathbb{R}^{n}\right)
\end{array}
$$

where the map ev $: C_{q} \times \operatorname{Imb}\left(S^{1}, \mathbb{R}^{n}\right) \rightarrow C_{q}\left(\mathbb{R}^{n}\right)$ is the evaluation map applied to $q$ distinct points in $S^{1}$ and $\hat{e} \hat{v}$ is its lift.

The diagram is commutative by construction. The main result is the following theorem proved in [9]:

Theorem 3.2 The spaces $C_{q, t}\left(\mathbb{R}^{n}\right)$ are smooth manifolds with corners. Moreover, the map $\hat{e v}$ and the projection $p_{1}$ (and, more generally, all projections $\left.C_{q, t}\left(\mathbb{R}^{n}\right) \rightarrow C_{q-k, t-l}\left(\mathbb{R}^{n}\right)\right)$ are smooth.

\subsection{Tautological forms}

It is not difficult to check that the maps $\phi_{i j}: C_{q}^{0}\left(\mathbb{R}^{n}\right) \rightarrow S^{n-1}$,

$$
\phi_{i j}\left(x_{1}, \ldots, x_{q}\right) \triangleq \frac{x_{j}-x_{i}}{\left|x_{j}-x_{i}\right|}
$$

extend to smooth maps on $C_{q}\left(\mathbb{R}^{n}\right)$. In fact, it is enough to consider the case $q=2$ and then apply Theorem 3.1.

Next we consider the so-called tautological forms, which are smooth as a consequence of Theorem 3.2. They are defined by (see [9]):

$$
\theta_{i j}\left(v^{n}\right) \triangleq \hat{e} \hat{v}^{*} \phi_{i j}^{*} v^{n} \in \Omega^{n-1}\left(C_{q, t}\left(\mathbb{R}^{n}\right)\right),
$$

where $v^{n}$ is a given normalized symmetric smooth top form on $S^{n-1}$.

Other forms on $C_{q, t}\left(\mathbb{R}^{n}\right)$ that we want to consider are obtained by pulling back the symmetric form $v^{n}$ via the map given by the combination of $p_{1}$ (s. (3.1)) with the map

$$
C_{q} \times \operatorname{Imb}\left(S^{1}, \mathbb{R}^{n}\right) \stackrel{p r_{i} \times i d}{\longrightarrow} S^{1} \times \operatorname{Imb}\left(S^{1}, \mathbb{R}^{n}\right) .
$$

where $p r_{i}: C_{q} \rightarrow S^{1}$ denotes the $i$ th projection. 
The pullback of forms on $S^{1} \times \operatorname{Imb}\left(S^{1}, \mathbb{R}^{n}\right)$ are forms on $C_{q, t}\left(\mathbb{R}^{n}\right)$. The main example that we have in mind is the "tangential tautological form"

$$
\theta_{i i}\left(v^{n}\right) \triangleq\left(e v_{i} \circ D\right)^{*} v^{n},
$$

where $D$ is the normalized derivative and $e v_{i}=e v \circ\left(p r_{i} \times i d\right)$.

\subsubsection{General properties of tautological forms}

Taking into account the definition of the maps $\phi_{i j}$ and of the tautological forms $(3.2,3.3)$, we have the following relations:

$$
\begin{aligned}
\theta_{i j}\left(v^{n}\right) & =(-1)^{n} \theta_{j i}\left(v^{n}\right), \quad i \neq j, \\
\theta_{i j}\left(v^{n}\right) \theta_{u v}\left(v^{n}\right) & =(-1)^{n+1} \theta_{u v}\left(v^{n}\right) \theta_{i j}\left(v^{n}\right), \\
\theta_{i j}^{2}\left(v^{n}\right) & =0 .
\end{aligned}
$$

The first relation is due to the action of the antipodal map on $S^{n-1}$, the second relation is a consequence of the degree of the tautological forms, and the third relation is an obvious consequence of the fact that the square of a top form is zero.

Finally, it may also be recalled that the cohomology classes of the tautological forms generate the whole cohomology of the configuration spaces of $\mathbb{R}^{n}$.

\subsection{Forms on the space of imbeddings}

In order to have differential forms on $\operatorname{Imb}\left(S^{1}, \mathbb{R}^{n}\right)$ we consider the "pushforward," or fiber-integration. For any bundle $(p: E \rightarrow B)$ such that the fiber $F$ is an $m$-dimensional compact oriented manifold (possibly with boundaries or corners), we define a map $p_{*}$ from the space of $(p+m)$-forms on $E$ to the space of $p$-forms on $B$, as follows:

$$
p_{*} \omega\left(X_{1}, \ldots, X_{m}\right) \triangleq \int_{F} \omega\left(\tilde{X}_{1}, \ldots, \tilde{X}_{m}, \cdot\right)
$$

where $\omega$ is a $(p+m)$-form on $E$ and $\tilde{X}_{i}$ is a vector field on $B$ whose projection yields the vector field $X_{i}$. The definition of $p_{*}$ is independent of the choice of the lifts $\tilde{X}_{i}$.

From the sequence of maps:

$$
\begin{gathered}
C_{q, t}\left(\mathbb{R}^{n}\right) \\
\downarrow p_{1} \\
C_{q} \times \operatorname{Imb}\left(S^{1}, \mathbb{R}^{n}\right) \\
\downarrow p_{2} \\
\operatorname{Imb}\left(S^{1}, \mathbb{R}^{n}\right)
\end{gathered}
$$

Algebraic 83 Geometric Topology, Volume 2 (2002) 
we obtain, by fiber-integrating products of $\theta_{i j}\left(v^{n}\right) \mathrm{s}$, forms on $\operatorname{Imb}\left(S^{1}, \mathbb{R}^{n}\right)$ which are not necessarily closed since the fiber is a manifold with corners. From the product of $k$ tautological forms we obtain a $((n-1) k-n t-q)$-form on $\operatorname{Imb}\left(S^{1}, \mathbb{R}^{n}\right)$.

Remark 3.3 Forms on $\operatorname{Imb}\left(S^{1}, \mathbb{R}^{n}\right)$ obtained this way are $\operatorname{Diff}^{+}\left(S^{1}\right)$-equivariant. Observe in fact that an orientation-preserving diffeomorphism of $S^{1}$ induces an orientation-preserving diffeomorphism of $C_{q}$. Horizontality follows then directly from the fiber integration along configuration spaces of $S^{1}$, while invariance is a consequence of the usual invariance of integrals under reparametrizations.

The exterior derivative of pushed-forward forms is given in terms of the generalized Stokes formula:

$$
d p_{*} \omega\left(X_{1}, \ldots, X_{m}\right)=p_{*} d \omega\left(X_{1}, \ldots, X_{m}\right)+(-1)^{\operatorname{deg} p_{*}^{\partial} \omega} p_{*}^{\partial} \omega\left(X_{1}, \ldots, X_{m}\right) .
$$

The coboundary operator $d$ on the l.h.s. refers to the space $\operatorname{Imb}\left(S^{1}, \mathbb{R}^{n}\right)$, while the coboundary operator on the r.h.s. refers to the space $C_{q, t}\left(\mathbb{R}^{n}\right)$. Moreover, $p_{*}^{\partial} \omega$ is given by

$$
p_{*}^{\partial} \omega(\gamma) \triangleq \int_{\partial C_{q, t}\left(\mathbb{R}^{n}, \gamma\right)} \omega
$$

where $\partial C_{q, t}\left(\mathbb{R}^{n}, \gamma\right)$ is the union of all the boundaries of codimension-1 of the fiber over the imbedding $\gamma$.

If we denote by $\lambda$ a product of tautological forms, then $d \lambda=0$. So we have

$$
d p_{*}(\lambda)=(-1)^{\operatorname{deg} p_{*}^{\partial} \lambda} p_{*}^{\partial}(\lambda) .
$$

In Appendix A we will consider these boundary push-forwards explicitly and show that, for $n>3$, only principal faces contribute.

Remark 3.4 Let us consider the $j$ th projection $p_{j}: C_{q}\left(\mathbb{R}^{n}\right) \rightarrow C_{q-1}\left(\mathbb{R}^{n}\right)$, and let us define

$$
\tau_{i k}=p_{j *} \theta_{i j}\left(v^{n}\right) \theta_{j k}\left(v^{n}\right) \in \Omega^{n-2}\left(C_{q-1}\left(\mathbb{R}^{n}\right)\right) .
$$

As a consequence of (3.4), (3.5) and (3.7), $\tau_{i k}$ is closed. But the $(n-2)$-nd cohomology group of $C_{q}\left(\mathbb{R}^{n}\right)$ is trivial. So the form $\tau_{i k}$ is exact.

Remark 3.5 Another particular case is the integral over $C_{q}\left(\mathbb{R}^{n}\right), n>3$, of a product of tautological forms with the condition that the situation of the preceding Remark never occurs (that is, we assume that for each point $i$, there 
are at least three tautological forms $\left.\theta_{i \bullet}\left(v^{n}\right)\right)$. In this case, the result must be a number, and this will not vanish only if the form degree matches the dimension of the space.

However, it is easy to prove that the form degree minus the dimension of the configuration space is always greater or equal to $(n-3) q / 2$. So these integrals always vanish.

\section{The complex of decorated graphs in any dimension}

Push-forwards of products of tautological forms along configuration spaces can be given a nice description in terms of graphs with a distinguished oriented loop. In the following, we will always represent the distinguished loop by a circle.

The idea is to represent each point in the configuration space as a vertex of a graph with the convention that all vertices constrained on the imbedding are put in order on the circle. Each tautological form will then be represented by an edge not belonging to the circle. (Actually, in the following we will reserve the term edge only to this kind of edges.)

In view of Remark 3.4, we can restrict ourselves to considering only graphs whose vertices not on the circle are at least trivalent. Moreover, thanks to Remark 3.5 and to (3.6), only connected graphs without multiple edges may yield nonzero results.

To keep track of the orientation of the configuration space and of the order in which one takes the product of tautological forms, the vertices and the edges must be numbered. Moreover, to distinguish between $\theta_{i j}\left(v^{n}\right)$ and $\theta_{j i}\left(v^{n}\right)$, one has to orient the edges.

However, thanks to the properties (3.4) and (3.5), the decoration of graphs can be simplified, as will be explained in subsection 4.1.

The differential of a form on $\operatorname{Imb}\left(S^{1}, \mathbb{R}^{n}\right)$ will be related by (3.7) to other push-forwards of products of tautological forms. As explained in Appendix A, also these push-forwards can be described in terms of graphs. As a consequence, we may relate the exterior derivative on the space of imbeddings to a certain coboundary operator on the complex of graphs. This is explained in subsection 4.2 .

The whole construction will finally be summarized in subsection 4.3, where we will also establish the relation between the graph cohomology and the de Rham cohomology of the space of imbeddings. 


\subsection{Decorated graphs in odd and even dimensions}

Following the above discussion, we will consider connected graphs consisting of an oriented circle and many edges joining vertices which may lie either on the circle (external vertices) or off the circle (internal vertices). We also require that each internal vertex should be at least trivalent.

In a graph we define a small loop to be an edge whose end-points are the same vertex. We call a small loop external or internal according to the nature of the corresponding vertex. (External small loops will represent forms $\theta_{i i}\left(v^{n}\right)$ as defined in (3.3), and internal small loops will be ruled away by (4.2).)

Next we assign a decoration to each graph in order to take into account the specific properties of the tautological forms:

- If $n$ is odd, then we label both internal and external vertices and assign an orientation (represented by an arrow) to each edge. We assume that the labelling of the external vertices is cyclic w.r.t. the orientation of the circle. Moreover, whenever we have an external small loop, we fix an ordering of the two half-edges that form it; notice that this ordering is chosen independently from the edge orientation.

- If $n$ is even, then the decoration consists in the labelling of the external vertices and of all the edges. Again we assume that the labelling of the external vertices is cyclic w.r.t. the orientation of the circle.

We now define $\mathcal{D}_{o}^{\prime}\left(\mathcal{D}_{e}^{\prime}\right)$ to be the real vector space generated by decorated graphs of odd (even) type (some examples of elements of these spaces are in figures 2, 3, 4 and 5).

As explained at the beginning of the Section, we actually do not need the whole spaces of graphs. We will restrict ourselves to the interesting spaces by dividing $\mathcal{D}_{o}^{\prime}$ and $\mathcal{D}_{e}^{\prime}$ by certain equivalence relations.

The first two relations do not depend on the decoration and are as follows:

$\Gamma \sim 0, \quad$ if two vertices in $\Gamma$ are joined by more than one edge,

$\Gamma \sim 0, \quad$ if $\Gamma$ contains an internal small loop.

(The first relation is motivated by (3.6), and the second by the fact that we cannot associate to an internal small loop any tautological form.)

Next, for any given pair of graphs $\widehat{\Gamma}$ and $\Gamma$ that differ only for the decoration, we introduce the following equivalence relations: 
- For $\Gamma, \widehat{\Gamma} \in \mathcal{D}_{o}^{\prime}$,

$$
\Gamma \sim(-1)^{\pi_{1}+\pi_{2}+l+s} \widehat{\Gamma},
$$

where $\pi_{1}$ is the order of the permutation of the internal vertices, $\pi_{2}$ is the order of the (cyclic) permutation of external vertices, $l$ is the number of edges whose orientation has been reversed, and $s$ is the number of external small loops on which the ordering of the half-edges has been reversed.

- For $\Gamma, \widehat{\Gamma} \in \mathcal{D}_{e}^{\prime}$,

$$
\Gamma \sim(-1)^{\pi+l} \widehat{\Gamma}
$$

where $\pi$ is the order of the (cyclic) permutation of the external vertices, and $l$ is the order of the permutation of the edges.

In order to have a well-defined, one-to-one correspondence between decorated graphs and the push-forwards of tautological forms as described at the beginning of the Section, we need to quotient $\mathcal{D}_{o}^{\prime}$ and $\mathcal{D}_{e}^{\prime}$ with respect to the equivalence relations $(4.1,4.2,4.3)$ and, respectively, by $(4.1,4.2,4.4)$. Namely, we define:

$$
\mathcal{D}_{o}:=\mathcal{D}_{o}^{\prime} / \sim \text { and } \mathcal{D}_{e}:=\mathcal{D}_{e}^{\prime} / \sim
$$

\subsubsection{Order and degree of decorated graphs}

The order of a graph $\Lambda$ (i.e., minus its Euler characteristic) is defined as

$$
\operatorname{ord} \Lambda=e-v_{i},
$$

where $e$ is the number of edges and $v_{i}$ is the number of internal vertices.

The degree of a graph $\Lambda$ is defined as

$$
\operatorname{deg} \Lambda=2 e-3 v_{i}-v_{e},
$$

where $e$ is the number of edges, $v_{e}$ is the number of external vertices and $v_{i}$ is the number of internal vertices.

In the particular case when the graph has only trivalent internal vertices and univalent external vertices, its degree is zero and its order is half the total number of vertices.

We consider $\mathcal{D}_{o}$ and $\mathcal{D}_{e}$ as graded vector spaces with respect to both the order and the degree.

We denote by $\mathcal{D}_{o}^{k, m}$ and $\mathcal{D}_{e}^{k, m}$ the equivalence classes of decorated graphs of order $k$ and degree $m$. 


\subsection{A coboundary operator for decorated graphs}

Now we want to introduce a coboundary operator on each space $\mathcal{D}_{o}$ and $\mathcal{D}_{e}$.

As explained at the beginning of this Section, we actually look for coboundary operators that, under the correspondence between graphs and configuration space integrals, are related to the exterior derivative on $\operatorname{Imb}\left(S^{1}, \mathbb{R}^{n}\right)$.

We will first define these operators on $\mathcal{D}_{o}^{\prime}$ and $\mathcal{D}_{e}^{\prime}$, and then prove that they descend to the quotients. These operators (both on the primed space and on their quotients) will be denoted by $\delta_{o}$ and $\delta_{e}$ respectively. When considering graphs of unspecified parity, we will simply use the symbol $\delta$.

First of all we introduce some terminology.

Definition 4.1 We call chord an edge whose end-points are distinct external vertices and short chord a chord whose end-points are consecutive vertices on the circle. We call regular edge an edge that is neither a chord nor a small loop. Finally we call arc a portion of the circle bounded by two consecutive external vertices.

For any graph $\Gamma, \delta \Gamma$ will be, by definition, a signed sum of decorated graphs obtained by contracting, one at a time, all the regular edges and all the arcs of $\Gamma$. Notice that the contraction of an arc joining the vertices of a short chord will produce an external small loop. In the odd case, we order its half-edges consistently with the orientation of the circle.

Edges and vertices are then relabelled as follows after contraction: if the new graph is obtained by contracting vertex $i$ with vertex $j$, then we relabel the vertices by lowering by one the labels of the vertices greater than $\max (i, j)$ and assign the label $\min (i, j)$ to the vertex where the contraction has happened. If we contract the edge $\alpha$, we lower by one the labels of all the edges greater than $\alpha$.

Moreover, we associate to each contraction a sign defined as follows:

- On the space $\mathcal{D}_{o}^{\prime}$, the sign associated to the contraction of the edge or of the arc joining the vertex $i$ to the vertex $j$ (using the orientation of the edge or the arc) is given by

$$
\sigma(i, j)= \begin{cases}(-1)^{j} & \text { if } j>i \\ (-1)^{i+1} & \text { if } j<i .\end{cases}
$$

Algebraic 83 Geometric Topology, Volume 2 (2002) 
- On the space $\mathcal{D}_{e}^{\prime}$, the sign associated to the contraction of the arc joining vertex $i$ to vertex $j$ is given by

$$
\sigma(i, j)= \begin{cases}(-1)^{j} & \text { if } j>i \\ (-1)^{i+1} & \text { if } j<i\end{cases}
$$

while, if we contract edge $\alpha$, we have the following sign:

$$
\sigma(\alpha)=(-1)^{\alpha+1+v_{e}},
$$

where $v_{e}$ is the number of external vertices.

The following Theorem shows that for both odd and even case, $\mathcal{D}$ is a complex of graded vector spaces.

Theorem 4.2 The operators $\delta_{o}$ and $\delta_{e}$ descend to $\mathcal{D}_{o}$ and, respectively, $\mathcal{D}_{e}$. They are both coboundary operators there, and we have

$$
\delta_{o}: \mathcal{D}_{o}^{k, m} \rightarrow \mathcal{D}_{o}^{k, m+1} \quad \delta_{e}: \mathcal{D}_{e}^{k, m} \rightarrow \mathcal{D}_{e}^{k, m+1} .
$$

The corresponding cohomology groups will be denoted in the following by $H^{k, m}\left(\mathcal{D}_{o}\right)$ and $H^{k, m}\left(\mathcal{D}_{e}\right)$.

Proof First we consider graphs of odd type and review the proof given in [7].

Let us consider the contraction of $(i j)$, i.e., of the edge or portion of circle between $i$ and $j$. If we exchange $i$ and $j$ or reverse an arrow, we get a minus sign; in both cases the roles of $i$ and $j$ are interchanged and we have $\sigma(i, j)=$ $-\sigma(j, i)$. Therefore $\delta_{o}$ is compatible with such an exchange.

Let us choose another vertex $k$, and exchange $j$ and $k$. We can assume $i<j$ and that $(i j)$ is oriented from $i$ to $j$. First we suppose $k>i$ and $k>j$. If we contract $(i j)$ we get a factor $(-1)^{j}$; if we exchange $j$ and $k$ and then contract $(i k)$ we get a factor $(-1)^{k+1}$. Obviously the underlying graph is the same in the two cases. We want to prove that the relabelling of one the two decorated graphs yields the other one, i.e., the two decorated graphs define the same element in $\mathcal{D}_{o}$. The indices lowered by one are, in the first case, all those greater than $j$ and in the second case all those greater than $k$. The set of vertices that, in the first case, are labelled as $j,(j+1), \cdots,(k-2),(k-1)$, are labelled, in the second case, as $(j+1),(j+2), \cdots,(k-1), j$ and the sign of the relevant permutation is $(-1)^{k-j+1}$.

In summary:

- if we contract $(i j)$ we get a sign $(-1)^{j}$; 
- if we swap $k$ and $j$, contract $(i k)$ and then relabel to get the same graph as in the previous case, we get the sign $(-1)^{k+1}(-1)^{k-j+1}=(-1)^{j}$.

Similarly, we can treat the case $k<i$. All other cases (contraction of $(i j)$ and swapping of $l$ with $k$ ) are trivial.

Now let us prove that $\delta_{o}^{2}=0$ by showing that contracting two different pairs $(\mathrm{ij})$ and $(r s)$ in opposite order yields the opposite sign.

If we have $i \neq r$ and $j \neq s$, then we can always assume that $i<j, r<s$ and $j<s$. Contracting $(i j)$ gives a sign $(-1)^{j}$ and lowers $s$ by one, so contracting $(r s)$ gives $(-1)^{s-1}$. If we contract $(r s)$ first, then $j$ is not lowered by one and the global sign is $(-1)^{s}(-1)^{j}$.

If $s=j$ and $i \neq r$, we can pass from a (double)-contraction to the other one by exchanging $i$ and $r$, with a change in sign in $\mathcal{D}_{o}$. The same holds for $s \neq j$ and $i=r$.

Next let us consider $\mathcal{D}_{e}^{\prime}$.

Again we have to show that a permutation of external vertices or of edges does not affect $\delta_{e}$. The case when we swap external vertices is identical to the case of $\mathcal{D}_{o}$, so we just have to verify what happens when we swap two edges labelled by $\alpha$ and $\beta$, with $\alpha<\beta$.

We claim that if we contract the edge $\alpha$ we obtain the same result as if we swap the edge $\alpha$ with the edge $\beta$ and subsequently contract the edge $\beta$. In fact in the first case the result is $(-1)^{\alpha+1+v_{e}}$ times a graph whose edges are: $(1, \ldots, \alpha-1, \alpha+1, \ldots, \beta-1, \beta, \beta+1, \ldots, t)$, while in the second case the result is $(-1)^{\beta+v_{e}}$ times a graph whose edges are $(1, \ldots, \alpha-1, \alpha+1, \ldots, \beta-$ $1, \alpha, \beta+1, \ldots, t)$. We now permute the labels of the edges of this last graph and obtain $(1, \ldots, \alpha-1, \alpha, \alpha+1, \ldots, \beta-1, \beta+1, \ldots, t)$; this permutation has order $(\beta-1)-(\alpha+1)+1=\beta-\alpha-1$. The total sign is therefore: $(-1)^{\beta+v_{e}}(-1)^{\beta-\alpha-1}=(-1)^{\alpha+1+v_{e}}$, i.e., the same result obtained by contracting the edge $\alpha$.

Finally, we have to show that $\delta_{e}^{2}=0$. As in the odd case, the proof consists in showing that if we make two contractions in different order, then we have opposite signs and hence the relevant graphs cancel.

This is obviously true if we contract two pairs of external vertices or two edges. Now we contract the arc between two consecutive external vertices (say $i$ and $j$, with $i<j$ ) and an edge $\alpha$. Remember that the number $v_{e}$ of external vertices appears in equation (4.9). If we contract $\alpha$ first, we do not change the labels of the external vertices and we get the global sign $(-1)^{\alpha+1+v_{e}}(-1)^{j}=$ 
$(-1)^{\alpha+1+v_{e}+j}$. If we contract $(i j)$ first, then the number of edges is lowered by one and so the global sign is $(-1)^{j}(-1)^{\alpha+1+\left(v_{e}-1\right)}=(-1)^{\alpha+v_{e}+j}$.

\subsection{The configuration space integral as a morphism of com- plexes}

We can finally give all the details of the construction described at the opening of the Section.

First of all, we fix $n>3$ and choose a form $v^{n}$ through which we define our tautological forms.

Definition 4.3 Let $\alpha$ be the antipodal map on $S^{n-1}$. We call symmetric form a normalized element $v^{n} \in \Omega^{n}\left(S^{n-1}\right)$ that satisfies $\alpha^{*} v^{n}=(-1)^{n} v^{n}$.

Obviously the standard volume form on $S^{n-1}$ is a symmetric form and, moreover, no nontrivial top form $w^{n}$ on $S^{n-1}$ can satisfy the condition $\alpha^{*} w^{n}=$ $(-1)^{n-1} w^{n}$.

From now on we will always assume that $v^{n}$ is a fixed normalized symmetric form on $S^{n-1}$.

Then we associate to every class of graphs $\Gamma$ a form $I\left(v^{n}\right)(\Gamma)$ on $\operatorname{Imb}\left(S^{1}, \mathbb{R}^{n}\right)$ in terms of a configuration space integral as follows:

- In the odd case, the edge joining the vertex $i$ to the vertex $j$ is replaced by the form $\theta_{i j}\left(v^{n}\right)$. The external small loop at $k$ is replaced by $(-1)^{l+s} \theta_{k k}\left(v^{n}\right)$, where $l=0$ if the edge orientation agrees with the ordering of the half-edges and $l=1$ otherwise, while $s=0$ if the ordering of the half-edges corresponds to the orientation of the circle and $s=1$ otherwise. Since all the forms are even, we do not have to say in which order we take their product. The orientation of the configuration space is determined by the numbering of the vertices.

- In the even case, we first have to choose a numbering of the internal vertices as well. Then the edge joining the vertex $i$ to the vertex $j$ is replaced by the form $\theta_{i j}\left(v^{n}\right)$, and an external small loop at $k$ is replaced by the form $\theta_{k k}\left(v^{n}\right)$. The numbering of the edges tells us in which order we have to take the product of tautological forms, while the numbering of the external vertices determines the orientation of the configuration space. (The numbering of internal vertices is on the other hand irrelevant.) 
We will denote by $I\left(v^{n}\right)$ the linear extension to $\mathcal{D}$ of the map just described. Then we have the following:

Theorem 4.4 For any $n>3$ and for any symmetric form $v^{n}$,

$$
\begin{array}{ll}
I\left(v^{n}\right):\left(\mathcal{D}_{o}^{k, m}, \delta_{o}\right) \longrightarrow\left(\Omega^{m+(n-3) k}\left(\operatorname{Imb}\left(S^{1}, \mathbb{R}^{n}\right)\right), d\right), & \text { if } n \text { is odd }, \\
I\left(v^{n}\right):\left(\mathcal{D}_{e}^{k, m}, \delta_{e}\right) \longrightarrow\left(\Omega^{m+(n-3) k}\left(\operatorname{Imb}\left(S^{1}, \mathbb{R}^{n}\right)\right), d\right), & \text { if } n \text { is even, }
\end{array}
$$

are chain maps.

Proof The coboundary operator $\delta$ has been defined in subsection 4.2 in such a way that it corresponds to the coboundary operator $d$ via Stokes' Theorem if one considers only principal faces (see Appendix A and in particular Thm. A.4 and Rem. A.5). The fact that $I$ is actually a chain map then follows from Theorem A.6 according to which we can neglect hidden faces.

As for the degree of these maps, this is easily computed: if the graph $\Lambda$ has $e$ edges, $v_{i}$ internal vertices and $v_{e}$ external vertices, then, by (4.6), $\operatorname{deg} \Lambda=$ $2 e-3 v_{i}-v_{e}$. On the other hand the degree of the corresponding differential form is

$$
\operatorname{deg} I(\Lambda)=(n-1) e-n v_{i}-v_{e}=\operatorname{deg} \Lambda+(n-3) \operatorname{ord} \Lambda,
$$

where ord $\Lambda$ is the order of the graph as defined in (4.5).

In the following we will denote also by $I\left(v_{n}\right)$ the map induced in cohomology:

$$
\begin{array}{ll}
I\left(v_{n}\right): H^{k, m}\left(\mathcal{D}_{o}\right) \longrightarrow H^{m+(n-3) k}\left(\operatorname{Imb}\left(S^{1}, \mathbb{R}^{n}\right)\right), & \text { if } n \text { is odd, } \\
I\left(v_{n}\right): H^{k, m}\left(\mathcal{D}_{e}\right) \longrightarrow H^{m+(n-3) k}\left(\operatorname{Imb}\left(S^{1}, \mathbb{R}^{n}\right)\right), & \text { if } n \text { is even. }
\end{array}
$$

The case $m=0$ is particularly interesting and will be discussed in the next section. In Section 6 we will prove that in this case the above homomorphisms are actually injective.

Recall finally that, as observed in Remark 3.3, the image of $I\left(v^{n}\right)$ lies in the subspace of $\operatorname{Diff}^{+}\left(S^{1}\right)$-equivariant forms. Thus, we can produce elements of the $\operatorname{Diff}^{+}\left(S^{1}\right)$-equivariant cohomology of $\operatorname{Imb}\left(S^{1}, \mathbb{R}^{n}\right)$.

\subsubsection{The dependency on the symmetric form $v^{n}$.}

We want now to consider the dependency of the chain map $I$ on the choice of the symmetric form. We have the following 
Proposition 4.5 Let $n>4$. If $v_{0}^{n}$ and $v_{1}^{n}$ are two symmetric forms and $\Gamma$ is a cocycle, then $I\left(v_{1}^{n}\right)(\Gamma)-I\left(v_{0}^{n}\right)(\Gamma)$ is an exact form.

Proof Let us write $v_{1}^{n}-v_{0}^{n}=d w^{n}$, where $w^{n} \in \Omega^{n-2}\left(S^{n-1}\right)$. We can assume that $\alpha_{n}^{*} w^{n}=(-1)^{n} w^{n}$. Then $v_{t}^{n}=v_{0}^{n}+t d w^{n}, t \in[0,1]$, interpolates between $v_{0}$ and $v_{1}$. We now define

$$
\tilde{v}^{n} \triangleq v_{0}^{n}+d\left(t w^{n}\right) \in \Omega^{n-1}\left(S^{n-1} \times[0,1]\right) .
$$

This form is still closed and symmetric: $\left(\alpha_{n} \times i d\right)^{*} \tilde{v}^{n}=(-1)^{n} \tilde{v}^{n}$.

Denoting by $i_{t}: S^{n-1} \hookrightarrow S^{n-1} \times[0,1]$ the inclusion at $t \in[0,1]$, we also have

$$
\begin{aligned}
i_{t}^{*} \tilde{v}^{n} & =v_{t}^{n}, \\
\int_{S^{n-1}} i_{t}^{*} \tilde{v}^{n} & =1 .
\end{aligned}
$$

Using this extended symmetric form, we can define extended tautological forms by

$$
\tilde{\theta}_{i j} \triangleq(\hat{e} \hat{v} \times i d)^{*}\left(\phi_{i j} \times i d\right)^{*} \tilde{v}^{n} \in \Omega^{n-1}\left(C_{q, t}\left(\mathbb{R}^{n}\right) \times[0,1]\right) .
$$

If we now replace the edges of a graph by these extended tautological forms, after integrating over the configuration space we will get a form on $\operatorname{Imb}\left(S^{1}, \mathbb{R}^{n}\right) \times$ $[0,1]$. Denote by $\tilde{I}\left(\tilde{v}^{n}\right)$ this map. Observe that, denoting by $j_{t}: \operatorname{Imb}\left(S^{1}, \mathbb{R}^{n}\right) \hookrightarrow$ $\operatorname{Imb}\left(S^{1}, \mathbb{R}^{n}\right) \times[0,1]$ the inclusion at $t$, we have

$$
j_{t}^{*} \tilde{I}\left(\tilde{v}^{n}\right)(\Gamma)=I\left(v_{t}^{n}\right)(\Gamma) .
$$

If $\Gamma$ is a cocycle, then the results of Appendix A (in particular Thms. A.4 and A.11) also show that $\tilde{I}\left(\tilde{v}^{n}\right)(\Gamma)$ is a closed form in $\Omega^{*}\left(\operatorname{Imb}\left(S^{1}, \mathbb{R}^{n}\right) \times[0,1]\right)$. As a consequence,

$$
I\left(v_{1}^{n}\right)(\Gamma)-I\left(v_{0}^{n}\right)(\Gamma)=d \pi_{*} \tilde{I}\left(\tilde{v}^{n}\right)(\Gamma),
$$

where $\pi$ is the projection $\operatorname{Imb}\left(S^{1}, \mathbb{R}^{n}\right) \times[0,1] \rightarrow \operatorname{Imb}\left(S^{1}, \mathbb{R}^{n}\right)$, and we have used again the generalized Stokes formula.

Thus, for $n>4$ the homomorphisms (4.10) and (4.11) do not depend on the choice of the symmetric form.

If $n=4$, then the homomorphisms might depend on the chosen symmetric form $v^{4}$ (see Rem. A.12).

Anyway, we will prove (see Thm. 6.4) that the integrals of $I\left(v^{n}\right)(\Gamma), \Gamma \in$ $H^{*, 0}(\mathcal{D})$, on the cycles given in $(2.4)$ do not depend on $v^{n}$, even for $n=4$.

Convention 4.6 In the rest of this paper, in all the tautological forms, we will omit the explicit dependency on the symmetric form $v^{n}$. 


\section{Cocycles of trivalent graphs}

Trivalent graphs are defined as (decorated) graphs having exactly one edge for each external vertex, while exactly three edges merge into each internal vertex. Notice that in particular trivalent graphs do not have external small loops. Moreover, trivalent graphs span $\mathcal{D}^{k, 0}$. Particular cases of trivalent graphs are of course chord diagrams.

We will look for cocycles that are linear combinations of trivalent graphs, that is, elements of $H^{k, 0}(\mathcal{D})$. We have the following

Proposition 5.1 If $\Gamma=\sum_{i} c_{i} \Gamma_{i} \in H^{k, 0}(\mathcal{D})$ is a cocycle given by a linear combination of trivalent graphs $\Gamma_{i}$, then at least one $\Gamma_{i}$ is a chord diagram. Moreover, no graph in $\Gamma$ may contain a short chord.

Proof Let $l$ be the minimum number of internal vertices among the graphs $\Gamma_{i}$. The first statement is equivalent to saying that $l=0$.

In fact, assume on the contrary that $l>0$, and let $\Gamma_{j}$ be a graph (which does not vanish in $\mathcal{D}$ ) with exactly $l$ internal vertices. Since we consider only connected graphs, there will be at least one internal vertex connected by one edge (call it $f$ ) to an external vertex.

In $\delta \Gamma_{j}$ there will then be a graph $\Gamma_{j}^{\prime}$ obtained by contracting the edge $f$.

First of all, notice that $\Gamma_{j}^{\prime}$ does not vanish in $\mathcal{D}$. In fact, if it did, then there would be an automorphism $\varphi$ of the graph underlying $\Gamma_{j}^{\prime}$ that would yield $-\Gamma_{j}^{\prime} \in \mathcal{D}$. Observe that the only 4 -valent vertex in $\Gamma_{j}^{\prime}$ has to be mapped to itself by $\varphi$. We can now extend this to an automorphism of $\Gamma_{j}$ by decollapsing $f$ after the application of $\varphi$ and deciding that $f$ is mapped into itself and each of its end-points are mapped into themselves (notice that we cannot interchange the end-points of $f$ since one is internal and the other is external). So by the extended automorphism we would prove that also $\Gamma_{j}$ vanishes in $\mathcal{D}$.

Since $\Gamma$ is a cocycle, there must be other graphs such that their images under the application of $\delta$ contain $\Gamma_{j}^{\prime}$. But there are only two possible graphs with this property, namely, those obtained by splitting the unique four-valent external vertex in $\Gamma_{j}^{\prime}$ in the two possible ways. But both these graphs have $l-1$ internal vertices, so $l$ cannot be the minimum.

To prove the second statement, observe first that $H^{1,0}(\mathcal{D})=0$ (in the odd case since the chord diagram is not closed, in the even case since the chord diagram vanishes by symmetry). 
So assume $k>1$. This means that the number of vertices is greater than 2 . Since we consider only connected graphs, this means that, if a graph $\Gamma_{i}$ contains a short chord, then there is only one arc (call it $a$ ) that has the same end-points as the short chord.

Let $\Gamma_{i}^{\prime}$ be the graph in $\delta \Gamma_{i}$ obtained by contracting $a$. Notice that $\Gamma_{i}^{\prime}$ contains an external small loop. Thus, since we do not allow internal small loops, there is no other graph whose image under the application of $\delta$ may contain $\Gamma_{i}^{\prime}$.

But as above we can prove that $\Gamma_{i}^{\prime}$ does not vanish (unless $\Gamma$ is zero itself.)

So $\Gamma$ cannot be a cocycle.

Applying the homomorphisms (4.10) and (4.11) to a trivalent cocycle $\Gamma$, we will then get cohomology classes on $\operatorname{Imb}\left(S^{1}, \mathbb{R}^{n}\right)$ of degree $(n-3)$ ord $\Gamma$.

The question whether nontrivial cocycles in the graph complex represent nontrivial cocycles in the cohomology ring $H^{*}\left(\operatorname{Imb}\left(S^{1}, \mathbb{R}^{n}\right)\right)$ will be addressed to in the next Section.

In the rest of this Section, we will discuss some examples of trivalent cocycles.

\subsection{The odd case}

Trivalent graphs have been widely studied $([5,23,9,6,1])$ in the case $n=3$. Here all graphs are associated to zero-forms, i.e., functions on $\left.\operatorname{Imb}\left(S^{1}, \mathbb{R}^{3}\right)\right)$ and, at each order, it is possible to find $\delta$-closed linear combinations of graphs.

Elements of $H^{0}\left(\operatorname{Imb}\left(S^{1}, \mathbb{R}^{3}\right)\right)$ are nothing but constant functions on connected components of the space $\operatorname{Imb}\left(S^{1}, \mathbb{R}^{3}\right)$, i.e., knot invariants. Such invariants are related to topological quantum field theories, namely, to the Chern-Simons theory [37] and to $B F$ theories (see [11] and references therein). Moreover, they are Vassiliev invariants (i.e., invariants of finite type) (see $[35,5,1])$.

When $n>3$, the homomorphism (4.10) implies that we can construct cohomology classes on $\operatorname{Imb}\left(S^{1}, \mathbb{R}^{n}\right)$ using exactly the same linear combinations of graphs that give knot invariants in three dimensions.

For instance the simplest cocycle

$$
1 / 4 \int_{C_{4}} \theta_{13} \theta_{24}-1 / 3 \int_{C_{3,1}} \theta_{14} \theta_{24} \theta_{34},
$$

see figure 2 , represents an element of $H^{2 n-6}\left(\operatorname{Imb}\left(S^{1}, \mathbb{R}^{n}\right)\right)$. 
At order 3, there is only one cocycle, which was calculated in [2] and [24] (s. also [5]). We show it in figure 3. In terms of integrals of tautological forms, it is given by:

$$
\begin{gathered}
\frac{1}{2} \int_{C_{4,2}} \theta_{15} \theta_{26} \theta_{36} \theta_{45} \theta_{56}+\frac{1}{3} \int_{C_{6}} \theta_{14} \theta_{25} \theta_{36}+\frac{1}{3} \int_{C_{3,3}} \theta_{14} \theta_{26} \theta_{35} \theta_{64} \theta_{65} \theta_{54}+ \\
-\int_{C_{5,1}} \theta_{16} \theta_{25} \theta_{36} \theta_{46}-\frac{1}{2} \int_{C_{6}} \theta_{14} \theta_{26} \theta_{35}+\frac{1}{2} \int_{C_{2,4}} \theta_{13} \theta_{25} \theta_{54} \theta_{56} \theta_{64} \theta_{63} \theta_{43} .
\end{gathered}
$$

Recall now that every Vassiliev invariant in three dimensions produces a non-

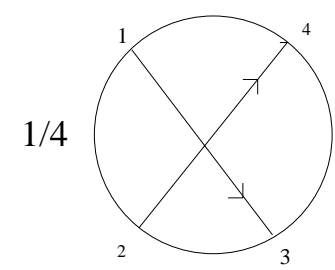

$-1 / 3$

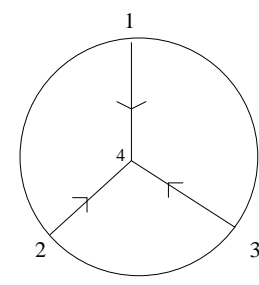

Figure 2: Cocycle of odd type at order 2
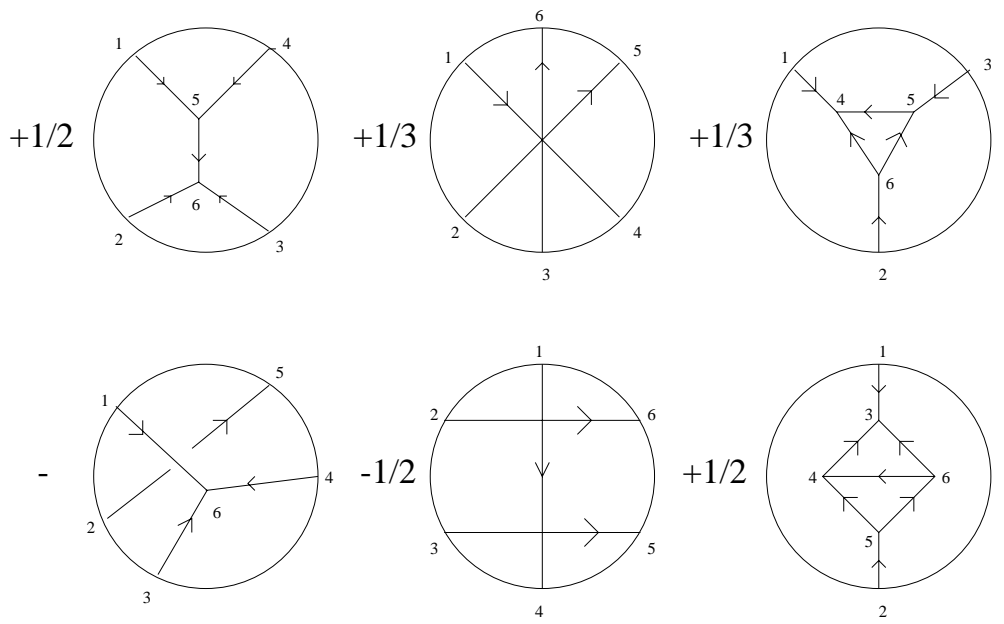

Figure 3: Cocycle of odd type at order 3

trivial cocycle of trivalent graphs of odd type [5]. It is also well known that there are nontrivial Vassiliev invariants at any order (e.g., coefficients of the Alexander-Conway or of the Jones polynomials). Hence, $H^{k, 0}\left(\mathcal{D}_{o}\right)$ contains nontrivial elements for any $k \geq 2$. 


\subsection{The even case}

For $n$ even, we use the homomorphism (4.11) to construct cohomology classes of $\operatorname{Imb}\left(S^{1}, \mathbb{R}^{n}\right)$.

It is easy to show that, at order two, there is only one cocycle in $\mathcal{D}_{e}$ (see figure 4), which induces, for every even $n$, the element

$$
1 / 4 \int_{C_{4}} \theta_{13} \theta_{24}-1 / 3 \int_{C_{3,1}} \theta_{14} \theta_{24} \theta_{34} \in H^{2 n-6}\left(\operatorname{Imb}\left(S^{1}, \mathbb{R}^{n}\right)\right) .
$$

At order 3 the vector space generated by trivalent graphs of even type has again dimension one, and the generator is given explicitly in figure 5 .

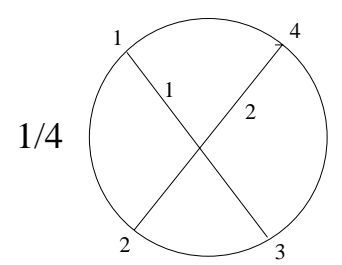

$-1 / 3$

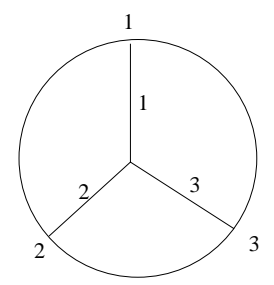

Figure 4: Cocycle of even type at order 2
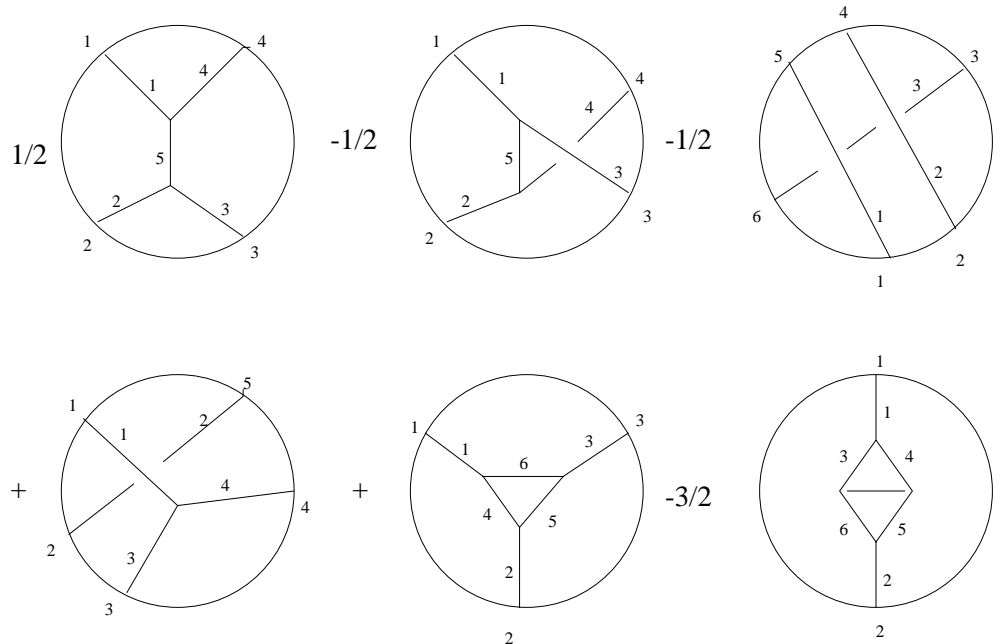

Figure 5: Cocycle of even type at order 3

Algebraic 63 Geometric Topology, Volume 2 (2002) 
The corresponding element of $H^{3 n-9}\left(\operatorname{Imb}\left(S^{1}, \mathbb{R}^{n}\right)\right)$ can be written as:

$$
\begin{aligned}
& \frac{1}{2} \int_{C_{4,2}} \theta_{1 a} \theta_{2 b} \theta_{3 b} \theta_{4 a} \theta_{a b}-\frac{1}{2} \int_{C_{4,2}} \theta_{1 a} \theta_{2 b} \theta_{3 a} \theta_{4 b} \theta_{a b}-\frac{1}{2} \int_{C_{6}} \theta_{15} \theta_{24} \theta_{36}+ \\
& +\int_{C_{5,1}} \theta_{1 a} \theta_{25} \theta_{3 a} \theta_{4 a}+\int_{C_{3,3}} \theta_{1 a} \theta_{2 b} \theta_{3 c} \theta_{a b} \theta_{b c} \theta_{c a}-\frac{3}{2} \int_{C_{2,4}} \theta_{1 a} \theta_{2 b} \theta_{a c} \theta_{a d} \theta_{b d} \theta_{b c} \theta_{c d} .
\end{aligned}
$$

Here we have labelled with numbers the external vertices and with letters the internal ones.

Moreover, we prove in [12] that, as in the odd case, there exist nontrivial cohomology classes of decorated trivalent graphs of even type in an infinite number of orders $k$.

\section{Trivalent graphs and nontrivial classes in $H^{*}\left(\operatorname{Imb}\left(S^{1}, \mathbb{R}^{n}\right)\right)$}

Let us now consider any nontrivial class in the cohomology of graphs. Under (4.10) or (4.11), this class will represent a possibly trivial cohomology class on $H^{*}\left(\operatorname{Imb}\left(S^{1}, \mathbb{R}^{n}\right)\right)$.

This Section is devoted to prove that, however, classes in the cohomology of trivalent graphs - that is, in $H^{*, 0}(\mathcal{D})$-yield nontrivial classes in the cohomology of $\operatorname{Imb}\left(S^{1}, \mathbb{R}^{n}\right)$.

The proof is based on the analysis of Section 2 and particularly on the criterion provided by Corollary 2.6.

The main result of the present Section is the following:

Theorem 6.1 Let $n>3$. An $[(n-3) k]$ th cohomology class on $\operatorname{Imb}\left(S^{1}, \mathbb{R}^{n}\right)$, corresponding via (4.10) or (4.11) to a linear combination of trivalent graphs, yields via (2.7) a nontrivial 0 th cohomology class on $\operatorname{Imm}_{k}^{\prime}\left(S^{1}, \mathbb{R}^{n}\right)$.

Proof Let $\Gamma=\sum_{i} \lambda_{i} \Gamma_{i} \in H^{k, 0}(\mathcal{D})$ be the given cocycle of trivalent graphs. Let us denote by $\omega\left(\omega_{i}\right)$ the image of $\Gamma\left(\Gamma_{i}\right)$ under (4.10) or (4.11). So $\omega=$ $\sum \lambda_{i} \omega_{i}$ is a closed $k(n-3)$-form on $\operatorname{Imb}\left(S^{1}, \mathbb{R}^{n}\right)$.

The forms $\omega_{i}$ are given by integrals of products of tautological forms over the corresponding configuration spaces $C_{q_{i}, t_{i}}\left(\mathbb{R}^{n}\right)$ with $q_{i} \leq 2 k$, and $q_{i}=2 k$ if and only if $\Gamma_{i}$ is one of the chord diagrams which are necessarily contained in $\Gamma$ by Prop. 5.1. 
We integrate first over the internal vertices and denote the result by $\mu_{i}$; then we have $\omega_{i}=\int_{C_{q_{i}}} \mu_{i}$.

We consider $\gamma \in \operatorname{Imm}_{k}^{\prime}\left(S^{1}, \mathbb{R}^{n}\right)$, and to each double point $p^{j}, j=1, \ldots, k$, of $\gamma$ we associate a ball $D_{j}$ of radius $\epsilon>0$. The intersection $\gamma \cap D_{j}$ is given by $l_{1}^{j}(\epsilon) \cup l_{2}^{j}(\epsilon)$, where $l_{a_{j}}^{j}(\epsilon), a_{j}=1,2$, are two closed segments as in figure 1 .

We now define $C_{q_{i}}^{\left(\epsilon ; j, a_{j}\right)}$ as the open subset of $C_{q_{i}}$ such that the image through $\gamma$ of all its projections over $S^{1}$ does not intersect $l_{a_{j}}^{j}(\epsilon)$. We also define

$$
C_{q_{i}}^{\epsilon} \triangleq \complement\left(\bigcup_{j=1}^{k} \bigcup_{a_{j}=1}^{2} C_{q_{i}}^{\left(\epsilon ; j, a_{j}\right)}\right)
$$

In other words, this complementary set $C_{q_{i}}^{\epsilon}$ is equal to the subset of $C_{q_{i}}$ for which all the projections over $S^{1}$ yield, through $\gamma$, one and only one element in each $l_{a_{j}}^{j}(\epsilon)$. Thus, $C_{q_{i}}^{\epsilon}=\emptyset$ unless $q_{i}=2 k$.

Next we define accordingly

$$
\begin{gathered}
\omega^{\epsilon} \triangleq \sum_{i} \lambda_{i} \int_{C_{q_{i}}^{\epsilon}} \mu_{i}, \\
\omega^{\left(\epsilon ; j, a_{j}\right)} \triangleq \sum_{i} \lambda_{i} \int_{C_{q_{i}}^{\left(\epsilon ; j, a_{j}\right)}} \mu_{i} .
\end{gathered}
$$

In the limit $\epsilon \rightarrow 0^{+}$, we recover the whole configuration space $C_{q_{i}}$, so $\omega^{\left(\epsilon ; j, a_{j}\right)}$ becomes $\omega$.

We now associate, once and for all, an index $a_{j} \in\{1,2\}$ to the $j$ th crossing and construct according to $(2.2)$ and $(2.4)$ a $k(n-3)$-cycle in $\operatorname{Imb}\left(S^{1}, \mathbb{R}^{n}\right)$ which we call $\beta(\epsilon, \delta)$, where $\epsilon$ and $\delta$ are small but positive. Different choices of the parameters $\epsilon$ and $\delta$ will yield homologous cycles in $\operatorname{Imb}\left(S^{1}, \mathbb{R}^{n}\right)$, as far as they do not become too large.

Any other choice of the indices $a_{j} \mathrm{~s}$ will also produce a homologous cycle in force of Proposition 2.2. We denote this new cycle by $\beta(\epsilon, \delta)+\partial \zeta\left(\epsilon, \delta ; j, a_{j}\right)$, for a suitable $(k(n-3)+1)$-chain $\zeta\left(\epsilon, \delta ; j, a_{j}\right)$.

We now want to compute the integral

$$
I\left(\epsilon, \delta, j, a_{j}\right)=\int_{\beta(\epsilon, \delta)} \omega^{\left(\epsilon ; j, a_{j}\right)}
$$

and show that

$$
\lim _{\delta \rightarrow 0^{+}} I\left(\epsilon, \delta, j, a_{j}\right)=0
$$

Algebraic 63 Geometric Topology, Volume 2 (2002) 
In fact, if in the explicit choice of $\beta(\epsilon, \delta)$ we have the same $a_{j}$ as in $\omega^{\left(\epsilon ; j, a_{j}\right)}$, then the above integral is zero for any $\delta$. Otherwise, it is equal to

$$
\int_{\zeta\left(\epsilon, \delta ; j, a_{j}\right)} d \omega^{\left(\epsilon ; j, a_{j}\right)}
$$

by Stokes' Theorem. The form $\omega^{\left(\epsilon ; j, a_{j}\right)}$ is not closed. But the main point here is that it is defined also on the space $\operatorname{Imm}_{1}^{\prime}\left(S^{1}, \mathbb{R}^{n}\right)(j) \subset \operatorname{Imm}_{1}\left(S^{1}, \mathbb{R}^{n}\right)$ given by the elements $\gamma$ for which $\gamma\left(t_{1}^{j}\right)=\gamma\left(t_{2}^{j}\right)$ is the only double point, and $t_{j}^{i} \neq 0$. This means that the above integral is well-defined also for $\delta=0$ where it vanishes by dimensional reasons. But this value is also equal to the limit $\delta \rightarrow 0^{+}$of the integral.

Next we consider all the sets $C_{q_{i}}^{\left(\epsilon ; j, a_{j}\right)}$ simultaneously. They are not disjoint, but we can redefine them (in an obvious way) so as to make them disjoint. With this we have

$$
\sum_{j} \int_{\beta(\epsilon, \delta)} \omega^{\left(\epsilon ; j, a_{j}\right)}+\int_{\beta(\epsilon, \delta)} \omega^{\epsilon}=\int_{\beta(\epsilon, \delta)} \omega .
$$

Observe now that this expression is independent of $\delta>0$ for $\delta$ small enough; in fact, different values of $\delta$ correspond to homologous cycles.

In particular, to compute the r.h.s. we can take the limit $\delta \rightarrow 0^{+}$. But in this limit the first term on the l.h.s. vanishes as proved above, and the integral of $\omega^{\epsilon}$ over $\beta(\epsilon, \delta)$ is like the integral of a Dirac-type current concentrated on the points of $C_{2 k}$ whose projections on $S^{1}$ yield exactly the set of those distinct points that are in pairs identified by $\gamma \in \operatorname{Imm}_{k}^{\prime}\left(S^{1}, \mathbb{R}^{n}\right)$.

Saying that only the forms on $C_{2 k}$ survive is tantamount as saying that only the chord diagrams contained in $\omega$ survive. Our final task is to prove that, when each chord connects two points on $S^{1}$ that are directly identified by $\gamma$ (i.e., that are in the pre-image of the same double point), then the corresponding integral is 1 , otherwise it is zero.

A chord connecting two (small) intervals that contain no other vertices can be seen as the $(n-3)$-form obtained by integrating $v^{n}$ over $I \times I$ with some identifications. To each point in $S^{n-3}$ we assign a way of lifting one of these two intervals and this generates a sphere $S^{n-2}$. The total integral associated to this chord will then be given by the linking number of this sphere $S^{n-2}$ with the other interval (seen as an indefinite line). This linking number is zero if the image of the two intervals does not contain the double point and, in force of Proposition 2.1, is 1 otherwise. 
Remark 6.2 Observe that for $n>4$ no care has to be taken in the actual choice of the symmetric form $v^{n}$, as follows from Prop. 4.5. If $n=4$, then different choices of $v^{n}$ might yield different homomorphisms from the graph cohomology to the de Rham cohomology of $\operatorname{Imb}\left(S^{1}, \mathbb{R}^{4}\right)$. However, the proof of the above proposition shows that the evaluation of $I\left(v^{4}\right)(\Gamma)$, for $\Gamma \in H^{k, 0}\left(\mathcal{D}_{e}\right)$, on the subspace of $H_{k(n-3)}\left(\operatorname{Imb}\left(S^{1}, \mathbb{R}^{4}\right)\right)$ spanned by the cycles defined in $(2.4)$ are independent of $v^{4}$.

Remark 6.3 The above proof can also be applied to the case $n=3$ assuming that one has already made the corrections for the anomalies appearing in that case. Notice that this proof does not reduce to the original one given in [1] from which it was however inspired - and seems to be a little easier.

It is clear from the proof of Theorem 6.1 that the image through (2.7) of the differential form $\omega=I\left(v^{n}\right)(\Lambda)$ associated to a trivalent cocycle $\Lambda$, is equivariant in the sense of Remark 2.7. Hence, we can recast the results of the previous proof as follows:

Theorem 6.4 Let $n>3$ and let

$$
\omega=I\left(v^{n}\right)(\Lambda) \in H^{k(n-3)}\left(\operatorname{Imb}\left(S^{1}, \mathbb{R}^{n}\right)\right),
$$

with $\Lambda \in H^{k, 0}(\mathcal{D})$ a linear combination of trivalent graphs. Then the blowup map (2.10) associates to $\omega$ the linear combinations of the chord diagrams contained in $\Lambda$, interpreted as an element of $H_{0}\left(\operatorname{Imm}_{k}\left(S^{1}, \mathbb{R}^{n}\right)\right)$. So the induced map $H^{k, 0}(\mathcal{D}) \rightarrow H^{0}\left(\operatorname{Imm}_{k}\left(S^{1}, \mathbb{R}^{n}\right)\right)$ is injective and independent of the choice of the symmetric form $v^{n}$.

We finally remark that the proof of Theorem 6.1 also implies the following result: a cycle of imbeddings of $S^{1}$ into $\mathbb{R}^{n}$ defined by a special immersion is nontrivial if there is a graph cocycle in which the chord diagram corresponding to the special immersion appears with nonzero coefficient. We do not know however under which conditions a chord diagram (with no separating chords) may be part of a graph cocycle. We will see in the next Section that, in the case of framed imbeddings (and for $n$ odd), the situation improves and, in particular, Theorem 1.3 holds.

\subsection{Proof of Theorem 1.1 and Corollary 1.2}

The statement about the chain maps, has been already proved in Thm. 4.4. The other statement is a direct consequence of the above Theorem, of Proposition 5.1 
and of Corollary 2.6. As for Corollary 1.2, we simply recall from subsections 5.1 and 5.2 that, irrespectively of the parity, there exist cocycles of trivalent graphs in an infinite number of orders.

\section{$7 \quad$ Framed imbeddings}

Let $K: S^{1} \rightarrow \mathbb{R}^{n}$ be an immersion. Recall that an orthonormal framing of $K$ is a trivialization of the pulled-back bundle $K^{*} S O\left(\mathbb{R}^{n}\right)$, where $S O\left(\mathbb{R}^{n}\right) \simeq$ $\mathbb{R}^{n} \times S O(n)$ is the orthonormal frame bundle of $\mathbb{R}^{n}$. Equivalently we may view the framing as a map $w: S^{1} \rightarrow S O(n)$. An orthonormal framing $w$ is said to be adapted if, for every $s \in S^{1}$, the last column of $w(s)$ is equal to the normalized tangent vector $D K(s):=\dot{K}(s) /|\dot{K}(s)|$. Let $p$ be the projection $S O(n) \rightarrow S^{n-1}$ in the quotient by $S O(n-1)$, viewed as the subgroup of $S O(n)$ fixing the vector $(0, \ldots, 0,1)$. Then saying that $w$ is a adapted framing is equivalent to stating that the following diagram commutes:

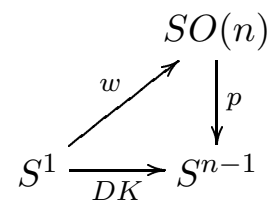

A pair $(K, w)$ consisting of an immersion $K$ and of a adapted framing $w$ will be shortly called a framed immersion. We will denote by $\operatorname{Imm}_{\mathrm{f}}\left(S^{1}, \mathbb{R}^{n}\right)$ the space of framed immersions and by $\operatorname{Imb}_{\mathrm{f}}\left(S^{1}, \mathbb{R}^{n}\right)$ its subspace of framed imbeddings.

By forgetting of the framing we get a map $\operatorname{Imb}_{\mathrm{f}}\left(S^{1}, \mathbb{R}^{n}\right) \rightarrow \operatorname{Imb}\left(S^{1}, \mathbb{R}^{n}\right)$; so we can pull back all cohomology classes constructed in the previous sections. Analogously, given a framed special immersion with $k$ transversal double points, we get a $k(n-3)$-cycle of framed imbeddings exactly by the same construction as in Section 2. We may repeat verbatim the proof of Theorem 6.1, so that Theorem 6.4 and 1.1 and Corollary 1.2 immediately generalize to the case of framed imbeddings. In the following we will show that, for $n$ odd, there are actually more cohomology classes on $\operatorname{Imb}_{\mathrm{f}}\left(S^{1}, \mathbb{R}^{n}\right)$ than on $\operatorname{Imb}\left(S^{1}, \mathbb{R}^{n}\right)$.

\subsection{Short chords and new cohomology classes on $\operatorname{Imb}_{\mathrm{f}}\left(S^{1}, \mathbb{R}^{2 s+1}\right)$}

We have seen in Section 4 that the boundary term corresponding to the contraction of a short chord - viz., an external small loop at a vertex labelled by, say, $k$-is the form $\theta_{k k}\left(v^{n}\right)$, i.e., the pullback of $v^{n}$ by using the composition 
of the projection to the $k$ th point on the circle with the normalized tangent vector map $D K$. We now wish to use a framing, and in particular diagram (7.1), to get rid of such terms. The plan is to find a form $\tau$ on $S O(n)$ with $d \tau^{n}=p^{*} v^{n}$.

Lemma 7.1 If $n$ is even, $\left[p^{*} v^{n}\right]$ is a nontrivial cohomology class on $S O(n)$. If $n$ is odd, $p^{*} v^{n}$ is exact.

Proof If $n=2 s$ then the restriction map $H^{*}(S O(2 s)) \rightarrow H^{*}(S O(2 s-1))$ is surjective and so, by Leray-Hirsch theorem, $H^{*}(S O(2 s))$ is a free module over $H^{*}\left(S^{2 s-1}\right)$, the action of the only non trivial generator of the cohomology of $S^{2 s-1}$ on $H^{*}\left(S^{2 s-1}\right)$ being the product by $\left[p^{*} v^{2 s}\right]$. Hence $p^{*} v^{2 s}$ cannot be exact. If $n=2 s+1$, then the class of the normalized top form $v^{2 s+1}$ of $S^{2 s}$ is half the Euler class and so $p^{*} v^{2 s+1}$ is exact.

Thus, we will have to restrict in the following to the case $n=2 s+1$. Using the framing, we see now that $\theta_{k k}\left(v^{2 s+1}\right)$ is exact; viz., it is equal to $d \vartheta_{k}\left(\tau^{2 s+1}\right)$, where $\vartheta_{k}\left(\tau^{2 s+1}\right)$ is the pullback of $\tau^{2 s+1}$ by the composition of the projection to the $k$ th point on the circle with the framing map $w$.

\subsubsection{Modified graph cohomology}

To keep track of the above forms, we modify the space of graphs $\mathcal{D}_{o}^{k, m}$ by introducing a new decoration, say a cross, that can be put on any external vertex (observe that a crossed vertex with no edges is allowed and that there can be more than one crossed vertex in the same graph). Crosses are numbered and a new equivalence relation $\Gamma \sim(-1)^{\sigma} \Gamma^{\prime}$ is introduced, where $\Gamma^{\prime}$ is the same graph as $\Gamma$ but for the numbering of crosses which is a permutation of the one in $\Gamma: \sigma$ is the order of this permutation. Let $x$ be the number of crosses in the graph $\Lambda$. We define the gradations

$$
\begin{aligned}
& \widetilde{\operatorname{ord} \Lambda}=e-v_{i}+x, \\
& \widetilde{\operatorname{deg} \Lambda}=2 e-3 v_{i}-v_{e}+x .
\end{aligned}
$$

We denote by $\widetilde{\mathcal{D}_{o}^{k, m}}$ the extended space of graphs of order $k$ and degree $m$. We also define a map

$$
\tilde{I}\left(v^{n}, \tau^{2 s+1}\right): \widetilde{\mathcal{D}_{o}^{k, m}} \rightarrow \Omega^{m+(2 s-2) k}\left(\operatorname{Imb}_{\mathrm{f}}\left(S^{1}, \mathbb{R}^{2 s+1}\right)\right),
$$


by the same rules as in subsection 4.3 plus a new rule that associates to a cross at the vertex $k$ the form $\vartheta_{k}\left(\tau^{2 s+1}\right.$ ) (the products of these odd forms being determined by the numbering of the crosses).

We also introduce a coboundary operator $\tilde{\delta}$ on $\widetilde{\mathcal{D}_{o}^{k, m}}$; it acts on all edges, arcs and crosses one at a time. Its action on edges and arcs is the same as for the coboundary operator $\delta$. Its action on the cross labelled $a$ at the vertex $i$ deletes the cross and produces an external small loop (with half-edges ordered consistently with the orientation of the circle); the sign associated to this operation on a graph $\Gamma$ is defined to be $(-1)^{\widetilde{\operatorname{deg}} \Gamma+a}$. Observe that $\tilde{\delta}$ raises $\widetilde{\operatorname{deg}}$ by 1 and leaves ord unchanged. It is not difficult to check that $\tilde{\delta}^{2}=0$.

It should be clear by now that the analogue of Theorem 4.4 for framed imbeddings is the following

\section{Proposition 7.2}

$$
\tilde{I}\left(v^{n}, \tau^{2 s+1}\right):\left(\widetilde{\mathcal{D}_{o}^{k, m}}, \tilde{\delta}\right) \rightarrow\left(\Omega^{m+(2 s-2) k}\left(\operatorname{Imb}_{\mathrm{f}}\left(S^{1}, \mathbb{R}^{2 s+1}\right)\right), d\right)
$$

is a chain map.

Proof The coboundary operator $\tilde{\delta}$ has been defined in such a way that $d \tilde{I}(\Gamma)$ is equal to $(-1)^{\operatorname{deg} \tilde{I}(\Gamma)+1} \tilde{I}(\tilde{\delta} \Gamma)$ if one neglects hidden faces. Thus, we have to prove that hidden faces do not contribute. But, since forms corresponding to crosses are basic in the fibrations $\Sigma_{r, \varsigma} \rightarrow C_{q-r+1, t-\varsigma}\left(\mathbb{R}^{2 s+1}\right)$, we may rely again on the results of Appendix A.

Finally, we notice that the proof of Theorem 6.1 generalizes immediately to the present case since the forms $\vartheta$ are well-defined on immersions. So we get the following generalization of Theorem 1.1:

Theorem 7.3 The induced homomorphism

$$
H_{\tilde{\delta}}^{k, 0}\left(\widetilde{\mathcal{D}_{o}}\right) \rightarrow H^{(2 s-2) k}\left(\operatorname{Imb}_{\mathrm{f}}\left(S^{1}, \mathbb{R}^{2 s+1}\right)\right)
$$

is injective.

\subsection{Relation between $H_{\tilde{\delta}}^{k, 0}\left(\widetilde{\mathcal{D}_{o}}\right)$ and $H_{\delta}^{k, 0}\left(\mathcal{D}_{o}\right)$}

First, we consider another graph complex, namely our old $\mathcal{D}_{o}^{k, m}$, but with a new coboundary operator $\underline{\delta}$ that differs from $\delta$ because it does not act on arcs 
joining the vertices of short chords. This new coboundary operator raises deg by 1 and leaves ord unchanged.

We define a map $\phi: \mathcal{D}_{o}^{k, m} \rightarrow \widetilde{\mathcal{D}_{o}^{k, m}}$. If $\Gamma$ does not contain short chords, we set $\phi(\Gamma)=\Gamma$. If instead $\Gamma$ contains a short chord, we proceed as follows: First we regard $\Gamma$ as an element of $\widetilde{\mathcal{D}_{o}^{k, m}}$. Then we pick a short chord (say, joining vertex $i$ to vertex $j$ ) and replace $\Gamma$ by $\Gamma-\sigma(i, j) \Gamma^{\prime}$, where $\Gamma^{\prime}$ is obtained by replacing the short chord by a crossed vertex with label $\min \{i, j\}$, while all other vertices are renumbered consequently. We repeat this for each short chord and number the crosses according to this order. Clearly $\phi$ is well-defined (i.e., it does not depend on the order in which we consider the short chords) and moreover we have the following

Proposition $7.4 \phi:\left(\mathcal{D}_{o}^{k, m}, \underline{\delta}\right) \rightarrow\left(\widetilde{\mathcal{D}_{o}^{k, m}}, \tilde{\delta}\right)$ is a chain map inducing an isomorphism:

$$
H^{k, 0}(\phi): H_{\underline{\delta}}^{k, 0}\left(\mathcal{D}_{o}\right) \stackrel{\sim}{\rightarrow} H_{\tilde{\delta}}^{k, 0}\left(\widetilde{\mathcal{D}_{o}}\right) .
$$

As a consequence we have:

Corollary 7.5 There is an injective homomorphism

$$
H_{\delta}^{k, 0}\left(\mathcal{D}_{o}\right) \hookrightarrow H_{\tilde{\delta}}^{k, 0}\left(\widetilde{\mathcal{D}_{o}}\right)
$$

Proof In Proposition 5.1 we showed that no element of $H_{\delta}^{k, 0}\left(\mathcal{D}_{o}\right)$ contains a graph with short chords. Since $\underline{\delta}$ is equal to $\delta$ on elements of $\mathcal{D}_{o}$ not containing short chords, we immediately deduce that $H_{\delta}^{k, 0}\left(\mathcal{D}_{o}\right) \subseteq H_{\underline{\delta}}^{k, 0}\left(\mathcal{D}_{o}\right) \cong H_{\tilde{\delta}}^{k, 0}\left(\widetilde{\mathcal{D}_{o}}\right)$.

\subsection{Relation between $H_{\tilde{\delta}}^{k, 0}\left(\widetilde{\mathcal{D}_{o}}\right)$ and Bar-Natan's algebra $\mathcal{A}_{k}(\bigcirc)$}

The advantage of dealing with the complex $\left(\mathcal{D}_{o}^{k, m}, \underline{\delta}\right)$ instead of $\left.\widetilde{\left(\mathcal{D}_{o}^{k, m}\right.}, \tilde{\delta}\right)$ is that $H_{\underline{\delta}}^{k, 0}\left(\mathcal{D}_{o}\right)$ is isomorphic to the dual of the space $\mathcal{A}_{k}(\bigcirc)$ of trivalent graphs with a distinguished circle and oriented vertices, modulo STU relations.

More precisely a $B N$ graph is defined as a connected graph made of a distinguished oriented circle and a certain number of edges, which are allowed to meet in two type of vertices: internal vertices in which three edges meet, and external vertices, in which an edge meets the distinguished circle. External vertices are oriented by one of the two possible cyclic orderings of the half edges emanating 
from that vertex. The degree of a BN graph is the number of its edges minus the number of internal vertices.

$\mathcal{D}(\bigcirc)$ is set to be the real vector space generated by $\mathrm{BN}$ graphs. $\mathcal{D}(\bigcirc)$ is graded by the degree of the graphs, $\mathcal{D}_{k}(\bigcirc)$ being the component of degree $k$.

Let $S T U$ be the subspace generated by the linear combinations of graphs as in figure 6 , where the undrawn part is the same for the three graphs that are considered.

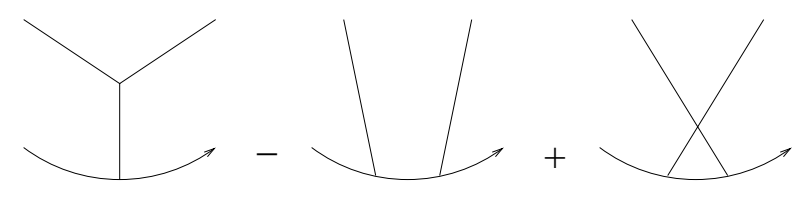

Figure 6: $S T U$ relation

We define $\mathcal{A}(\bigcirc) \triangleq \mathcal{D}(\bigcirc) / S T U$ and we denote its dual by $\mathcal{A}^{*}(\bigcirc)$. $\mathcal{A}(\bigcirc)$ is graded and we denote the $k$ th component by $\mathcal{A}_{k}(\bigcirc)$. The space $\mathcal{A}^{*}(\bigcirc)$ is also know as the space of weight systems.

Proposition 7.6 There is an isomorphism $\mathcal{A}_{k}^{*}(\bigcirc) \cong H_{\underline{\delta}}^{k, 0}\left(\mathcal{D}_{o}\right)$

The proof is given in Theorem 1 of ref. [1].

Remark 7.7 It can be shown, following the proof of Prop. 7.6, that given $\Gamma \in \mathcal{D}_{k}(\bigcirc)$ and $w \in \mathcal{A}_{k}^{*}(\bigcirc)$ such that $w(\Gamma) \neq 0$, then the image of $w$ in $H_{\underline{\delta}}^{k, 0}\left(\mathcal{D}_{o}\right)$, under the isomorphism of Prop. 7.6, is a cocycle containing a graph that, modulo decoration, is identical to $\Gamma$.

It is well known $[4,5]$ that any finite dimensional Lie algebra with an invariant inner product gives rise to a family of elements of $\mathcal{A}^{*}(\bigcirc)$. In particular it is possible to construct elements of $\mathcal{A}_{k}^{*}(\bigcirc)$ which are non zero on graphs containing short chords, and hence elements of $H_{\delta}^{k, 0}\left(\mathcal{D}_{o}\right)$ which contains graphs with short chords. This implies that $H_{\tilde{\delta}}^{k, 0}\left(\widetilde{\mathcal{D}_{o}}\right) \cong H_{\underline{\delta}}^{k, 0}\left(\mathcal{D}_{o}\right)$ is actually strictly bigger than $H_{\delta}^{k, 0}\left(\mathcal{D}_{o}\right)$.

\subsection{Proof of Theorem 1.3}

For every given chord diagram there exist cocycles of zero degree in $\left(\mathcal{D}_{o}^{k, m}, \hat{\delta}\right)$ containing it. This follows from Remark 7.7 and from the fact that the weight system determined by the Lie algebra $\mathfrak{g l}(n)$ is nonzero on every chord diagram. 


\section{On the cohomology of the spaces of imbeddings and immersions of $S^{1}$ into $\mathbb{R}^{n}$}

In this Section we will relate the cohomology of the spaces $\operatorname{Imb}\left(S^{1}, \mathbb{R}^{n}\right)$ and $\operatorname{Imm}\left(S^{1}, \mathbb{R}^{n}\right)$ of imbeddings and immersions of $S^{1}$ into $\mathbb{R}^{n}$. This will allow us to consider examples of nontrivial cohomology classes of $\operatorname{Imb}\left(S^{1}, \mathbb{R}^{n}\right)$ not coming from trivalent graphs and even not coming from our graphs. Moreover these last classes are not equivariant w.r.t. the action of $\operatorname{Diff}^{+}\left(S^{1}\right)$.

We will first review known facts about the computation of $H^{*}\left(\operatorname{Imm}\left(S^{1}, \mathbb{R}^{n}\right)\right)$ by Chen's iterated integrals, and then we will consider the restriction of the above cohomology classes to $\operatorname{Imb}\left(S^{1}, \mathbb{R}^{n}\right)$.

Given a Riemannian simply connected manifold $M$, we naturally associate to any $\psi \in \operatorname{Imm}\left(S^{1}, M\right)$ its normalized derivative $D \psi$, which is an element of the loop space $\mathcal{L} S M$, where $S M$ denotes the tangent sphere bundle. We denote by $\mathcal{L} X$ the free loop space over a manifold $X$.

Smale proved [31] (see also [10]) the following:

Theorem 8.1 The map $D: \operatorname{Imm}\left(S^{1}, M\right) \rightarrow \mathcal{L} S M$ is a weak homotopy equivalence.

In particular the space $\operatorname{Imm}\left(S^{1}, \mathbb{R}^{n}\right)$ is weakly homotopy equivalent to $\mathcal{L} S^{n-1}$.

The cohomology of the loop space of a simply connected manifold $X$ can be computed via Chen's iterated integrals [18] (for a purely algebraic approach see, for example,[28]).

Let us consider the $m$-dimensional simplex $\Sigma_{m}:=\left\{\left(t_{1}, \ldots t_{m}\right) \in \mathbb{R}^{m}: 0 \leq\right.$ $\left.t_{1} \leq \ldots \leq t_{m} \leq 1\right\}$ and the map $\Phi$

$$
\Phi: \begin{gathered}
\Sigma_{m} \times \mathcal{L} X \\
\left(\left(t_{1}, \ldots, t_{m}\right), \gamma\right)
\end{gathered} \quad \longrightarrow \quad \begin{gathered}
X \times \cdots \times X \\
\left(\gamma\left(t_{1}\right), \ldots, \gamma\left(t_{m}\right)\right) .
\end{gathered}
$$

From a collection of forms $w_{1}, \ldots, w_{m}$ in $\Omega^{*}(X)$, we obtain the form $p r_{1}^{*} w_{1} \wedge$, $\ldots, \wedge p r_{m}^{*} w_{m}$ on $X^{m}$, where $p r_{i}: X^{m} \rightarrow X$ denotes the projection onto the $i$ th factor. The iterated Chen integral is defined by pullback via the map $\Phi$ and subsequent integration on $\Sigma_{m}$. The result is a form on $\mathcal{L} X$,

Moreover, any form $w_{0} \in \Omega^{*}(X)$ defines also a form of the same degree on $\mathcal{L} X$, denoted again as $w_{0}$, obtained by pullback via the evaluation map at the initial 
point of the loop. In summary, we consider the following class of forms on $\mathcal{L} X$ with degree $\sum_{i=0}^{m} \operatorname{deg}\left(w_{i}\right)-m$ :

$$
w_{0} P\left(w_{1}, \cdots, w_{m}\right) \triangleq w_{0} \int_{\Sigma_{m}} \Phi^{*}\left(p r_{1}^{*} w_{1} \wedge, \ldots \wedge p r_{m}^{*} w_{m}\right) .
$$

When all the forms $w_{i}$ are equal to a given form $w$, we use the shorter notation $w_{0} P_{m}(w)$.

When $M=\mathbb{R}^{n}$, it is sufficient to consider $w_{i}=v^{n}$, where $v^{n}$ is a fixed normalized top form on $S^{n-1}$. In fact, we have [21]:

Proposition 8.2 The algebra $H^{*}\left(\mathcal{L} S^{n-1}, \mathbb{R}\right)$ is the associative and graded commutative algebra with the following set of generators and relations:

- For $n$ odd, the generators are $P_{1}\left(v^{n}\right) P_{2 s}\left(v^{n}\right)$ in degree $\left.(2 s+1)(n-2)\right)$ and $v^{n} P_{2 s}\left(v^{n}\right)$ in degree $(2 s+1)(n-2)+1$, with $s=0,1, \ldots$ The relations are given by the requirement that the product of any two generators is zero.

- For $n$ even, the generators are $v^{n}$ in degree $n-1$ and $P_{1}\left(v^{n}\right)$ in degree $n-2$ with the only relation $\left(v^{n}\right)^{2}=0$.

This determines uniquely the real cohomology of $\operatorname{Imm}\left(S^{1}, \mathbb{R}^{n}\right)$, which is obtained by pulling back to $\operatorname{Imm}\left(S^{1}, \mathbb{R}^{n}\right)$ the above classes via the map $D$ (normalized derivative).

Some of the above cohomology classes are equivariant with respect to the action of the group $\operatorname{Diff}^{+}\left(S^{1}\right)$ of orientation preserving diffeomorphisms of $S^{1}$, on $\operatorname{Imm}\left(S^{1}, \mathbb{R}^{n}\right)$. More precisely, one can prove [21] that irrespectively of the dimension $n$, the forms $v^{n} P_{k}\left(v^{n}\right)$ do not yield equivariant classes on $\operatorname{Imm}\left(S^{1}, \mathbb{R}^{n}\right)$ and that the equivariant generators are given by $D^{*} P_{1}\left(v^{n}\right)$ for $n$ even and by $D^{*} P_{1}\left(v^{n}\right) D^{*} P_{2 s}\left(v_{n}\right)$, with relations as before, for $n$ odd.

Remark 8.3 The equivariant forms $D^{*} P_{k}\left(v^{n}\right)$ can be constructed using the morphisms $I\left(v^{n}\right)$ of equations (4.10) and (4.11). Let us consider in fact a graph $\Gamma_{k} \in \mathcal{D}^{k, k}$ consisting of $k$ small loops attached to $k$ different external vertices. Since $\Gamma_{k}$ contains no chord, $I\left(v^{n}\right)\left(\Gamma_{k}\right)$ is a well defined differential form on $\operatorname{Imm}\left(S^{1}, \mathbb{R}^{n}\right)$, and we have an (obvious) identification $D^{*} P_{k}\left(v^{n}\right)=I\left(v^{n}\right)\left(\Gamma_{k}\right)$. For instance, the closure of $D^{*} P_{k}\left(v^{n}\right)$ can be seen as a consequence of the equation $\delta \Gamma_{k}=0$, which holds both in the odd and in the even case.

We now consider the restriction of the above forms and classes on $\operatorname{Imm}\left(S^{1}, \mathbb{R}^{n}\right)$ to forms and classes on $\operatorname{Imb}\left(S^{1}, \mathbb{R}^{n}\right)$. It is convenient to assume that $v^{n}$ is a 
symmetric form (see Def 4.3). Then we define $\theta\left(v^{n}\right)$ to be the configuration space integral $I\left(v^{n}\right)\left(\Gamma_{1}^{\prime}\right)$ where $\Gamma_{1}^{\prime} \in \mathcal{D}^{1,0}$ is the graph with two external vertices joined by one chord, and $I\left(v^{n}\right)$ the homomorphisms of eq. (4.10) or (4.11), depending on the parity of $n$. Clearly $\theta\left(v^{n}\right)$ is zero if $n$ is even because the corresponding graph is zero by the relation (4.4). On the contrary, if $n$ is odd, one has the following:

Lemma 8.4 If $n$ is odd, we have

$$
d \theta\left(v^{n}\right)=-2 D^{*} P_{1}\left(v^{n}\right) .
$$

Proof First, let us write

$$
\theta\left(v^{n}\right)=\int_{S^{1} \times I} \phi^{*} v^{n}
$$

where

$$
\begin{array}{ccc}
\phi: S^{1} \times I \times \operatorname{Imb}\left(S^{1}, \mathbb{R}^{n}\right) & \rightarrow & S^{n-1} \\
(s, t, \psi) & \mapsto \frac{\psi(s+t)-\psi(s)}{\|\psi(s+t)-\psi(s)\|,}
\end{array}
$$

We have

$$
d \theta\left(v^{n}\right)=\int_{S^{1} \times \partial I} i^{*} \phi^{*} v^{n}=D^{*} P_{1}\left(\alpha^{*} v^{n}\right)-D^{*} P_{1}\left(v^{n}\right),
$$

where the map $i$ denotes the restriction to the boundary $S^{1} \times \partial I$ and $\alpha$ is the antipodal map on $S^{n-1}$.

We now prove the following:

Theorem 8.5 If $n$ is odd, then the inclusion of $\operatorname{Imb}\left(S^{1}, \mathbb{R}^{n}\right)$ in $\operatorname{Imm}\left(S^{1}, \mathbb{R}^{n}\right)$ induces the zero map in (real) cohomology.

Proof The restriction of $D^{*} P_{1}\left(v^{n}\right)$ to $\operatorname{Imb}\left(S^{1}, \mathbb{R}^{n}\right)$ is exact in force of Lemma 8.4. When we restrict the generators $D^{*}\left[P_{1}\left(v^{n}\right) P_{2 s}\left(v^{n}\right)\right]$ in degree $(2 s+1)(n-$ $2)$ ) to $\operatorname{Imb}\left(S^{1}, \mathbb{R}^{n}\right)$, we obtain trivial cocycles, as shown in the following equation:

$$
\begin{aligned}
& -2 D^{*}\left[P_{1}\left(v^{n}\right) P_{2 s}\left(v^{n}\right)\right]= \\
& \quad=d\left(\theta\left(v^{n}\right) D^{*} P_{2 s}\left(v^{n}\right)-(1 / 2) \theta^{2}\left(v^{n}\right) D^{*}\left[v^{n} P_{2 s-2}\left(v^{n}\right)\right]\right) .
\end{aligned}
$$

Next we prove that also the generators $D^{*}\left[v^{n} P_{2 s}\left(v^{n}\right)\right]$ represent trivial cocycles when restricted to $\operatorname{Imb}\left(S^{1}, \mathbb{R}^{n}\right)$. 
First we notice that $D^{*} v^{n}$ is exact when restricted to $\operatorname{Imb}\left(S^{1}, \mathbb{R}^{n}\right)$. Consider in fact the map $\widehat{\phi}: I \times \operatorname{Imb}\left(S^{1}, \mathbb{R}^{n}\right) \rightarrow S^{n-1}$ given by the restriction of the map $\phi$ of equation (8.2) to $\{0\} \times I \times \operatorname{Imb}\left(S^{1}, \mathbb{R}^{n}\right) \subset S^{1} \times I \times \operatorname{Imb}\left(S^{1}, \mathbb{R}^{n}\right)$ and define

$$
\Theta\left(v^{n}\right) \triangleq \int_{I} \widehat{\phi}^{*} v^{n}
$$

We conclude that

$$
d \Theta\left(v^{n}\right)=-2 D^{*} v^{n} .
$$

Finally, we have

$$
-2 D^{*}\left[v^{n} P_{2 s}\left(v^{n}\right)\right]=d\left(\Theta\left(v^{n}\right) D^{*} P_{2 s}\left(v^{n}\right)-(1 / 2) \Theta^{2}\left(v^{n}\right) D^{*} P_{2 s-1}\left(v^{n}\right)\right) .
$$

Thanks to the identification $D^{*} P_{k}\left(v^{n}\right)=I\left(v^{n}\right)\left(\Gamma_{k}\right)$ of Remark 8.3, Lemma 8.4 and equation (8.4) can be seen in graph cohomology as the existence of $\Gamma_{k}^{\prime} \in$ $\mathcal{D}_{o}^{k, k-1}$ such that $\delta_{o}\left(\Gamma_{k}^{\prime}\right)=\Gamma_{k}$.

Theorem 8.5 has been proved in [9], but only for the case $H^{1}\left(\operatorname{Imm}\left(S^{1}, \mathbb{R}^{3}\right)\right)$.

If $n$ is even, then we know that $\theta\left(v^{n}\right)$ is zero and moreover it is easy to see that $\Theta\left(v^{n}\right)$ (as defined in (8.5)) is closed. Thus, if $n$ is even, we cannot conclude anymore that the inclusion $\operatorname{Imb}\left(S^{1}, \mathbb{R}^{n}\right) \hookrightarrow \operatorname{Imm}\left(S^{1}, \mathbb{R}^{n}\right)$ induces the zero morphism in cohomology. On the contrary, we find that the classes $D^{*} P_{1}\left(v^{n}\right)$, $D^{*} v^{n}$ and $D^{*}\left[v^{n} P_{1}\left(v^{n}\right)\right]$ remain nontrivial when restricted to $\operatorname{Imb}\left(S^{1}, \mathbb{R}^{n}\right)$. We first have:

Proposition 8.6 If $n$ is even, then the restriction of $D^{*}\left[v^{n} P_{1}\left(v^{n}\right)\right]$ to $\operatorname{Imb}\left(S^{1}, \mathbb{R}^{n}\right)$ is a nontrivial cocycle of degree $2 n-3$.

Proof Let us consider the real Stiefel manifold

$$
V_{n, 2}=S O(n) / S O(n-2) .
$$

It is an orientable compact manifold of dimension $(2 n-3)$. To each $(\mathbf{x}, \mathbf{y}) \in V_{n, 2}$ we associate the following element of $\operatorname{Imb}\left(S^{1}, \mathbb{R}^{n}\right)$ :

$$
t \mapsto((1-\cos t) \mathbf{x}+\sin t \mathbf{y}) .
$$

The integral of the cocycle $D^{*}\left[v^{n} P_{1}\left(v^{n}\right)\right]$ over the image of the map (8.6) is given by the degree of the map:

$$
\begin{aligned}
& f: S^{1} \times V_{n, 2}(\mathbb{R}) \rightarrow S^{n-1} \times S^{n-1} \\
& (t, \mathbf{x}, \mathbf{y}) \mapsto(\mathbf{y}, \sin t \mathbf{x}+\cos t \mathbf{y})
\end{aligned}
$$

Algebraic 83 Geometric Topology, Volume 2 (2002) 
It is evident that

$$
\begin{aligned}
f^{-1}((0, \cdots, 0,0,1),(0, \cdots, 0,1,0)) & = \\
=(\pi / 2,(0, \cdots, 0,1,0),(0, \cdots, 0,0,1)) \cup & \cup(3 \pi / 2,(0, \cdots, 0,-1,0),(0, \cdots, 0,0,1))
\end{aligned}
$$

and

$$
\operatorname{deg} f=1+(-1)^{n}
$$

Corollary 8.7 If $n$ is even, then the restrictions of $D^{*} P_{1}\left(v^{n}\right)$ and $D^{*} v^{n}$ to $\operatorname{Imb}\left(S^{1}, \mathbb{R}^{n}\right)$ are nontrivial cocycles of degree $n-2$ and, respectively, $n-1$.

Proof $D^{*}\left[v^{n} P_{1}\left(v^{n}\right)\right]$ is the product of $D^{*} v^{n}$ and $D^{*} P_{1}\left(v^{n}\right)$.

For completeness, we explicitly construct examples of cycles of $\operatorname{Imb}\left(S^{1}, \mathbb{R}^{n}\right)$ below, nontrivially paired with the above cocycles.

If $n$ is even, then a nontrivial element $\Gamma_{n-2} \in H_{n-2}\left(\operatorname{Imb}\left(S^{1}, \mathbb{R}^{n}\right)\right)$ is given by the map that assigns to each normalized vector $\mathbf{x} \in S^{n-2}$, represented as $\left(x_{1}, x_{2}, \cdots, x_{n-1}, 0\right) \in \mathbb{R}^{n}$, the imbedded loop in $\mathbb{R}^{n}$ given by

$$
t \mapsto\left(x_{1}(1-\cos t), x_{2}(1-\cos t), \ldots, x_{n-1}(1-\cos t), \sin t\right) .
$$

By computing the degree of the normalized derivative of this map, it turns out that the evaluation of $D^{*} P_{1}\left(v^{n}\right)$ on $\Gamma_{n-2}$ is -2 .

Again, for $n=2 s$, a nontrivial element $\Gamma_{n-1} \in H_{n-1}\left(\operatorname{Imb}\left(S^{1}, \mathbb{R}^{n}\right)\right)$ is given by the map that assigns to each normalized vector

$$
\mathbf{x}=\left(x_{1}, y_{1}, x_{2}, y_{2}, \cdots, x_{s}, y_{s}\right) \in S^{2 s-1} \subset \mathbb{R}^{2 s}
$$

the loop based at $\mathbf{x}$ given by

$$
\begin{gathered}
t \mapsto\left(x_{1}(1-\cos t)+y_{1} \sin t,-x_{1} \sin t+y_{1}(1-\cos t), \cdots\right. \\
\left.\cdots, x_{s}(1-\cos t)+y_{s} \sin t,-x_{s} \sin t+y_{s}(1-\cos t)\right),
\end{gathered}
$$

and the evaluation of $D^{*} v^{n}$ on $\Gamma_{n-1}$ yields $(-1)^{s}$.

Remark 8.8 Corollary 8.7 extends Thm. 1.1 since it provides an example in which the morphism (4.11) is nontrivial on a nontrivalent graph cocycle $\Gamma_{1} \in H^{1,1}\left(\mathcal{D}_{e}\right)$. 
Remark 8.9 To complete the discussion on the nature of the restriction to $\operatorname{Imb}\left(S^{1}, \mathbb{R}^{n}\right)$ of nontrivial classes on $\operatorname{Imm}\left(S^{1}, \mathbb{R}^{n}\right)$, we should also say something about $D^{*} P_{k}\left(v^{n}\right)$ and $D^{*}\left[v^{n} P_{k}\left(v^{n}\right)\right]$ when $n$ is even and $k>1$.

At the moment, however, we have no cycles to pair with them in order to prove that they are nonzero nor have an argument to prove their vanishing. We may only comment that these classes are images under (4.11) of nontrivial cocycles of graphs (in the sense of Section 4). Therefore, triviality of the above classes would imply that (4.11) is not injective.

Remark 8.10 We have chosen $v^{n}$ to be a given symmetric form. Notice however that the main results of this Section (Theorem 8.5, Proposition 8.6 and Corollary 8.7) are independent of the choice of the volume form on $S^{n-1}$ ).

\section{A Appendix: Integrals along the boundary and van- ishing theorems}

The goal of this Appendix is to show that, as long as $n>3$ and we use only symmetric forms, hidden faces do not contribute to Stokes' theorem.

\section{A.1 Codimension-one faces}

Let us begin with a description of the codimension-one faces of $\partial C_{q, t}\left(\mathbb{R}^{n}\right)$ These faces can be divided into three classes:

Type I $s \geq 2$ of the points in $\mathbb{R}^{n}$ collapse together;

Type II $s \geq 1$ of the points in $\mathbb{R}^{n}$ escape together to infinity;

Type III $r \geq 1$ of the points in $S^{1}$ and $s \geq 0$ of the points in $\mathbb{R}^{n}$, with $r+s \geq 2$, collapse together; we denote these faces by the symbol $\Sigma_{r, s}$.

Boundary faces of type I or type II are given by $\widehat{C}_{s}\left(\mathbb{R}^{n}\right) \times C_{q, t-s+1}\left(\mathbb{R}^{n}\right)$ and $\widehat{C}_{s+1}\left(\mathbb{R}^{n}\right)$ $\times C_{q, t-s}\left(\mathbb{R}^{n}\right)$ respectively. Here $\widehat{C}_{k}\left(\mathbb{R}^{n}\right)$ is obtained from $\left(\mathbb{R}^{n}\right)^{k} / G-G$ being the group of global translations and scalings - by blowing up all diagonals. Each $\widehat{C}_{k}\left(\mathbb{R}^{n}\right)$ is a compact manifold with corners. In the simplest case, $k=2$, we have $\widehat{C}_{2}\left(\mathbb{R}^{n}\right)=S^{n-1}$. Notice however that in the following we will only need to consider the interior of $\widehat{C}_{k}\left(\mathbb{R}^{n}\right)$ which is easily identified with $C_{k-1}^{0}\left(\mathbb{R}^{n}\right) / \mathbb{R}_{+}$, where the group $\mathbb{R}_{+}$acts by rescaling all components.

A boundary face $\Sigma_{r, 0}$ is a fibration over $C_{q-r+1, t}\left(\mathbb{R}^{n}\right)$ got by pulling back $\widehat{C}_{r}\left(T S^{1}\right)$. In the case when $s>0$, the description of $\Sigma_{r, s}$ is a little bit longer. Actually, we will 
only describe the interior of $\Sigma_{r, s}$ (which is enough for Stokes' theorem). Let us first define

$$
\begin{array}{r}
S^{n-1} \ltimes \mathcal{C}_{r, s} \triangleq\left\{a \in S^{n-1},\left(x_{1}, \ldots, x_{r}\right) \in \mathbb{R}^{r},\left(y_{1}, \ldots, y_{s}\right) \in\left(\mathbb{R}^{n}\right)^{s} \mid\right. \\
\left.a\left(x_{i}\right) \neq y_{j}, \forall i, j ; x_{i}=x_{j} \Rightarrow i=j ; y_{i}=y_{j} \Rightarrow i=j\right\},
\end{array}
$$

where $a \in S^{n-1}$ is seen as a linear map $\mathbb{R} \rightarrow \mathbb{R}^{n}$.

Next introduce the equivalence relations

$$
\begin{aligned}
\left(a ; x_{1}, \ldots ; y_{1}, \ldots\right) & \sim\left(a ; \lambda x_{1}, \ldots ; \lambda y_{1}, \ldots\right) \\
\left(a ; x_{1}, \ldots ; y_{1}, \ldots\right) & \sim\left(a ; x_{1}+\xi, \ldots ; y_{1}+a(\xi), \ldots\right)
\end{aligned}
$$

with $\lambda>0$ and $\xi \in \mathbb{R}$, and denote by $S^{n-1} \ltimes \widehat{C}_{r, s}$ the quotient. This is a fibration over $S^{n-1}$ whose fiber will be denoted by $\widehat{C}_{r, s}$.

Then the interior $\operatorname{int}\left(\Sigma_{r, s}\right)$ of a type III face, corresponding to the collapse of $r$ points on $S^{1}$ together with $s$ points in $\mathbb{R}^{n}$, is a fibration over $C_{q-r+1, t-s}\left(\mathbb{R}^{n}\right)$ obtained by pulling back $\left(S^{n-1} \ltimes \widehat{C}_{r, s}\right)$ via the composition $\widehat{D}$ of the normalized derivative $D: \operatorname{Imb}\left(S^{1}, \mathbb{R}^{n}\right) \rightarrow \mathcal{L} S^{n-1}$ with the evaluation at the position on $S^{1}$ where the points have collapsed:

$$
\begin{array}{ccc}
\operatorname{int}\left(\Sigma_{r, s}\right) & \longrightarrow & S^{n-1} \ltimes \widehat{C}_{r, s} \\
\downarrow & & \downarrow \\
C_{q-r+1, t-s}\left(\mathbb{R}^{n}\right) & \stackrel{\widehat{D}}{\longrightarrow} & S^{n-1}
\end{array}
$$

Remark A.1 What is really important for all the subsequent considerations are the dimensions of the fibers of the above spaces. A simple computation yields

$$
\begin{aligned}
& d_{0, s} \doteq \operatorname{dim} \widehat{C}_{s}\left(\mathbb{R}^{n}\right)=n s-n-1, \\
& d_{r, s} \doteq \operatorname{dim} \widehat{C}_{r, s}=r+n s-2 \text { for } r>0 .
\end{aligned}
$$

We end with the following

Definition A.2 A codimension-one face is called a principal face if it is of type I with $s=2$, or of type II with $s=1$, or of type III with $r+s=2$. All other codimension-one faces are called hidden faces.

\section{A.2 Subgraphs corresponding to codimension-one faces}

In Section 4, we have explained how to associate integrals of tautological forms over configuration spaces to decorated graphs. Here we want to represent the integration along codimension-one faces in terms of subgraphs.

First we introduce the notion of decorated graphs with a point at infinity. These are just decorated graphs with one extra vertex labelled by $\infty$ and the condition that $\infty$ is 
not the end-point of any edge. Of course, there is a one-to-one correspondence $\Gamma \mapsto \tilde{\Gamma}$ between decorated graphs and decorated graphs with a point at infinity.

A subgraph $\Gamma^{\prime}$ of a graph $\tilde{\Gamma}$ is a graph whose vertices are a subset of the vertices in $\tilde{\Gamma}$ and whose edges and arcs are the subset of the edges and arcs of $\tilde{\Gamma}$ whose end-points are distinct and both in the subset of vertices of $\Gamma^{\prime}$. (Notice in particular that an external small loop based at a vertex in $\Gamma^{\prime}$ is not considered as an edge of $\Gamma^{\prime}$.)

A subgraph $\Gamma^{\prime}$ of a decorated graph with a point at infinity is said to be admissible if one of the following conditions is fulfilled:

Type I $\Gamma^{\prime}$ has $s \geq 2$ internal vertices and no other vertices;

Type II $\Gamma^{\prime}$ has $s \geq 1$ internal vertices plus the $\infty$-vertex;

Type III $\Gamma^{\prime}$ has $r \geq 1$ external vertices and $s \geq 0$ internal vertices, with $r+s \geq 2$.

The correspondence between admissible subgraphs and codimension-one faces should be clear.

Definition A.3 An admissible subgraph will be called principal (hidden) if the corresponding face is principal (hidden).

If $\Gamma^{\prime}$ is an admissible subgraph of $\Gamma$, we define the reduction of $\Gamma$ modulo $\Gamma^{\prime}$, which we will denote by $\left[\Gamma / \Gamma^{\prime}\right]$, as follows. First we define two vertices in $\Gamma$ to be equivalent if they both belong to $\Gamma^{\prime}$. Then the set of vertices of $\left[\Gamma / \Gamma^{\prime}\right]$ is the set of vertices of $\Gamma$ modulo the equivalence relations, while the set of edges and arcs contains all the edges and arcs of $\Gamma$ that do not belong to $\Gamma^{\prime}$. The vertex in $\left[\Gamma / \Gamma^{\prime}\right]$ whose pre-image in $\Gamma$ is $\Gamma^{\prime}$ will be denoted by $v\left(\Gamma^{\prime}, \Gamma\right)$.

Given any graph $\Gamma$, we will denote by $\alpha_{\Gamma}$ the corresponding form (product of tautological forms associated to the edges).

Following the description of the preceding Section, we can compute the form on the space of imbeddings obtained by integrating $\alpha_{\Gamma}$ along the codimension-one face corresponding to $\Gamma^{\prime}$ as follows: we integrate along the configuration space corresponding to $\left[\Gamma / \Gamma^{\prime}\right]$ the product of $\alpha_{\left[\Gamma / \Gamma^{\prime}\right]}$ and a form which we denote by $\alpha\left(\Gamma / \Gamma^{\prime}\right)$.

For faces of type I, $\alpha\left(\Gamma / \Gamma^{\prime}\right)$ is the form $I\left(\Gamma^{\prime}\right)$ obtained by integrating $\alpha_{\Gamma^{\prime}}$ along $\Gamma^{\prime}$, and as such vanishes unless it is a zero-form.

For faces of type II, sending a subgraph $\Gamma^{\prime}$ to infinity is, roughly speaking, like shrinking to a point the complementary subgraph. More precisely, we consider the subgraph $\widetilde{\Gamma}$ of $\Gamma$ consisting of the vertices which are not in $\Gamma^{\prime}$, and of those arcs and edges whose end-points do not belong to $\Gamma^{\prime}$. Then we form the quotient $\Gamma^{\prime \prime}=\Gamma / \widetilde{\Gamma}$ and denote by $v$ the vertex of $\Gamma^{\prime \prime}$ whose pre-image is $\widetilde{\Gamma}$. Now $\alpha\left(\Gamma / \Gamma^{\prime}\right)$ is obtained by integrating $\alpha_{\Gamma^{\prime \prime}}$ over the subspace of the configuration space corresponding to $\Gamma^{\prime \prime}$, in which $v$ is fixed to $0 \in \mathbb{R}^{n}$. Again, we have that $\alpha\left(\Gamma / \Gamma^{\prime}\right)$ vanishes unless it is a zero-form.

For a face of type III, $\alpha\left(\Gamma / \Gamma^{\prime}\right)$ is the pullback of $I\left(\Gamma^{\prime}\right)$ via the map $D$ (normalized derivative), combined with the evaluation at the point corresponding to the vertex $v\left(\Gamma^{\prime}, \Gamma\right)$. As a consequence, $I\left(\Gamma^{\prime}\right)$ and $\alpha\left(\Gamma / \Gamma^{\prime}\right)$ vanish unless their degree is less or equal to $n-1$. 


\section{A.3 Vanishing theorems}

Now we can state the following:

Theorem A.4 If $\Gamma^{\prime}$ is a principal subgraph, then $I\left(\Gamma^{\prime}\right)$ vanishes unless

(1) $\Gamma^{\prime}$ is of type $I$ and contains exactly one edge, or

(2) $\Gamma^{\prime}$ is of type III with $r=s=1$ and contains exactly one edge, or

(3) $\Gamma^{\prime}$ is of type III with $r=2$ and has at most one edge.

In the first two cases or in the third case with no edges $I\left(\Gamma^{\prime}\right)= \pm 1$. In the third case with one edge, $I\left(\Gamma^{\prime}\right)= \pm v^{n}$.

Proof Since $\Gamma^{\prime}$ is principal, it contains at most two vertices. Thus, by our definition of graphs, there is at most one edge in $\Gamma^{\prime}$ if it of type I or III, and no edge if $\Gamma^{\prime}$ is of type II.

If there are no edges, then there is nothing to be integrated. However, the fiber corresponding to $\Gamma^{\prime}$ has dimension strictly positive (equal to $n-1$ ) if it is of type I or II, or if it is of type III with $r=s=1$.

If however the face is of type III with $r=2$ the fiber dimension is zero, and we get $I\left(\Gamma^{\prime}\right)=1$

If there is exactly one edge and $\Gamma^{\prime}$ is of type I or it is of type III with $r=s=1$, we have to integrate the $(n-1)$-dimensional form $v$ over the fiber which is $S^{n-1}$. This yields $I\left(\Gamma^{\prime}\right)= \pm 1$.

If $\Gamma^{\prime}$ is of type III with $r=2$ and contains exactly one edge (to which corresponds $\left.v^{n}\right)$, then the fiber is zero-dimensional, and $I\left(\Gamma^{\prime}\right)= \pm v^{n}$.

Remark A.5 The cases in which $I\left(\Gamma^{\prime}\right)$ does not vanish correspond exactly to the integrations over principal faces considered in Section 4. The sign $I\left(\Gamma^{\prime}\right)= \pm 1$ is the sign $\sigma$ of (4.7), (4.8) and (4.9), i.e., the sign associated to the operator $\delta$; the case $I\left(\Gamma^{\prime}\right)= \pm v^{n}$ corresponds to the contraction of an arc joining the end-points of a short chord.

As for the hidden faces, we have the following result, whose proof is contained in the next subsubsection.

Theorem A.6 If $\Gamma^{\prime}$ is an admissible subgraph corresponding to a hidden face and $n>3$, then $\alpha\left(\Gamma / \Gamma^{\prime}\right)=0$. 


\section{A.3.1 Proof of Theorem A.6}

We follow the strategy proposed in [26] and [32].

Throughout this subsection, by valence of a vertex we mean the number of edges ending at that vertex.

We start by proving the following basic facts.

Lemma A.7 If $\Gamma^{\prime}$ is hidden and contains a zero-valent vertex different from $\infty$, then $\alpha\left(\Gamma / \Gamma^{\prime}\right)=0$.

Lemma A.8 If $\Gamma^{\prime}$ is hidden and contains a univalent internal vertex, then $\alpha\left(\Gamma / \Gamma^{\prime}\right)=$ 0 .

Lemma A.9 If $\Gamma^{\prime}$ is hidden and contains a bivalent internal vertex, then $\alpha\left(\Gamma / \Gamma^{\prime}\right)=$ 0 .

Proof of Lemma A.7 Let $i$ be the 0 -valent vertex and $x_{i}$ the corresponding point. We perform the $x_{i}$-integration first. Let us compute the dimension of this fiber. Since $\Gamma^{\prime}$ is hidden, we can use the other points to fix translations and scalings (or just scalings if $\Gamma^{\prime}$ is of type II). So the fiber is $n$-dimensional if $i$ is internal and 1 -dimensional if $i$ is external.

The form to be integrated over this fiber is however of degree zero since no edge ends at $i$.

Proof of Lemma A.8 As in the previous proof, we perform the $x_{i}$-integration first, where $i$ is an internal univalent vertex now. As before, the fiber is $n$-dimensional.

The form to be integrated over this fiber is the tautological form corresponding to the edge ending at $i$. Thus, it has degree $n-1$.

Proof of Lemma A.9 Now we perform the $n$-dimensional integration over $x_{i}$, where $i$ is an internal bivalent vertex.

Let $j$ and $k$ be the end-points of the two edges starting at $i$. If $j=k$, then the integrand form is $\theta_{i j}^{2}=0$.

So assume $j \neq k$. The $x_{i}$-integration can be the extended to $\mathbb{R}^{n}$ with only $x_{j}$ and $x_{k}$ blown up. Let us denote by $C\left(\mathbb{R}^{n} ; x_{j}, x_{k}\right)$ this space. We must then compute

$$
\alpha=\int_{C\left(\mathbb{R}^{n} ; x_{j}, x_{k}\right)} \theta_{i j} \theta_{i k} .
$$

Algebraic 83 Geometric Topology, Volume 2 (2002) 
Following Kontsevich [26], we consider the involution $\chi$ of $C\left(\mathbb{R}^{n} ; x_{j}, x_{k}\right)$ that maps $x_{i}$ to $x_{j}+x_{k}-x_{i}$. Observe that $\chi$ is orientation preserving (reversing) if $n$ is even (odd). Moreover,

$$
\begin{aligned}
\chi^{*} \theta_{i j} & =\theta_{k i}=(-1)^{n} \theta_{i k}, \\
\chi^{*} \theta_{i k} & =\theta_{j i}=(-1)^{n} \theta_{i j} .
\end{aligned}
$$

Thus,

$$
\alpha=(-1)^{n} \int_{C\left(\mathbb{R}^{n} ; x_{j}, x_{k}\right)} \theta_{i k} \theta_{i j}=(-1)^{n}(-1)^{n-1} \int_{C\left(\mathbb{R}^{n} ; x_{j}, x_{k}\right)} \theta_{i j} \theta_{i k}=-\alpha .
$$

Therefore, $\alpha=0$.

Thanks to the above Lemmas, we can from now on assume that all internal vertices in $\Gamma^{\prime}$ are at least trivalent and that all external vertices are at least univalent. As a consequence, we have

$$
2 e^{\prime} \geq r+3 s
$$

where $e^{\prime}$ is the number of edges in $\Gamma^{\prime}, r$ is the number of external vertices (possibly zero) and $s$ that of internal vertices. Therefore, since any edge carries an $(n-1)$-form,

$$
\operatorname{deg} \alpha\left(\Gamma / \Gamma^{\prime}\right) \geq \begin{cases}(n-1) \frac{r+3 s}{2}-d_{r, s} & \text { if } \Gamma^{\prime} \text { is of type I or III, } \\ (n-1) \frac{3(s+1)}{2}-d_{0, s+1} & \text { if } \Gamma^{\prime} \text { is of type II }\end{cases}
$$

with $d_{r, s}$ defined in (A.1). In order to prove that $\alpha\left(\Gamma / \Gamma^{\prime}\right)$ vanishes, it is then enough to check that $\operatorname{deg} \alpha\left(\Gamma / \Gamma^{\prime}\right)>0$ for $\Gamma^{\prime}$ of type I or II, and that $\operatorname{deg} \alpha\left(\Gamma / \Gamma^{\prime}\right)>n-1$ for $\Gamma^{\prime}$ of type III (otherwise the form to be integrated over the fiber vanishes by dimensional reasons). Finally, a straightforward computation yields

$$
\operatorname{deg} \alpha\left(\Gamma / \Gamma^{\prime}\right) \geq \begin{cases}\frac{(n-3) s}{2}+n+1 & \text { if } \Gamma^{\prime} \text { is of type I, } \\ \frac{(n-3) s}{2}+\frac{5 n-1}{2} & \text { if } \Gamma^{\prime} \text { is of type II } \\ \frac{(n-3)(r+s-2)}{2}+n-1 & \text { if } \Gamma^{\prime} \text { is of type III. }\end{cases}
$$

The result now follows from the facts that $\Gamma^{\prime}$ is hidden (so $r+s>2$ ) and that $n>3$.

Remark A.10 In the case $n=3$, the previous argument fails for $\Gamma^{\prime}$ of type III, and suitable corrections have to be found (see $[9,32,29]$ ).

In the case $n=2$, the argument fails in general and more refined techniques have to be considered (see [27]).

Algebraic $6 \mathcal{G}$ Geometric $\mathcal{T}$ opology, Volume 2 (2002) 


\section{A.4 Forms depending on a parameter}

As in the proof of Prop. 4.5, we now consider tautological forms that are pullbacks of a form $\tilde{v}^{n}$ in $\Omega^{n-1}\left(S^{n-1} \times[0,1]\right)$ which is closed and symmetric and integrates to one on the sphere for any value of the parameter $t \in[0,1]$. The corresponding configuration space integral $\tilde{I}(\Gamma)$ yields then a form on $\operatorname{Imb}\left(S^{1}, \mathbb{R}^{n}\right) \times[0,1]$. It is not difficult to check that Thm. A.4 still holds with $I$ replaced by $\tilde{I}$. Moreover, we have the following generalization of Thm. A.6:

Theorem A.11 If $\Gamma^{\prime}$ is an admissible subgraph corresponding to a hidden face and $n>4$, then $\tilde{I}\left(\Gamma^{\prime}\right)=0$.

Proof We proceed as in the proof of Thm. A.6 till the paragraph just before (A.2).

Now we observe that, by dimensional reasons, $\tilde{I}\left(\Gamma^{\prime}\right)$ vanishes if $\operatorname{deg} \tilde{I}\left(\Gamma^{\prime}\right)>1$ for $\Gamma^{\prime}$ of type I or II, or $\operatorname{deg} \tilde{I}\left(\Gamma^{\prime}\right)>n$ for $\Gamma^{\prime}$ of type III.

But these inequalities are again satisfied by (A.2).

Remark A.12 In the case $n=4$, the above result might not hold. For example, consider the 5-dimensional face $\Sigma_{3,1}$ and assume that in $\Gamma^{\prime}$ there are three edges (so, a 9 -form). After integration, we are left with a 4 -form on $S^{3} \times[0,1]$, and there is no apparent reason why it should vanish.

In particular, consider the cocycle of figure 4. When all the vertices in the second graph collapse, we are exactly in the same situation as above. Since there are no other edges, we can now perform the integration over $[0,1]$. We are finally left with the integral along the knot of the pullback via the normalized derivative of a top form $w^{4}$ on $S^{3}$. Thus, on the r.h.s. of (4.12), we must add a multiple (possibly zero) of $P_{1}\left(w^{4}\right)$, that is, of the lowest cohomology class obtained by restriction from the space of immersions.

\section{References}

[1] D. Altschuler, L. Freidel: "Vassiliev knot invariants and Chern-Simons perturbation theory to all orders," Commun. Math. Phys. 187, 261-287 (1997)

[2] M. Alvarez, J. M. F. Labastida: "Analysis of observables in Chern-Simons perturbation theory," Nucl. Phys. B 395, 198 (1993); and "Numerical knot invariants of finite type from Chern-Simons perturbation theory," Nucl. Phys. B 433, 555 (1995)

[3] S. Axelrod, I. M. Singer: "Chern-Simons perturbation theory," in Proceedings of the XXth DGM Conference, edited by S. Catto and A. Rocha (World Scientific, Singapore, 1992), pp. 3-45; "Chern-Simons perturbation theory. II," J. Diff. Geom. 39, 173-213 (1994)

[4] D. Bar-Natan: Perturbative Aspects of Chern-Simons Topological Quantum Field Theory, Ph.D. thesis, Princeton University, (1991); "Perturbative ChernSimons theory," J. of Knot Theory and its Ramifications 4, 503-548 (1995) 
[5] D. Bar-Natan: "On the Vassiliev knot invariants," Topology 34, 423-472 (1995)

[6] R. Bott: "Configuration spaces and imbedding invariants," Turkish J. Math. 20, no. 1, 1-17 (1996)

[7] R. Bott, A. S. Cattaneo: "Integral invariants of 3-manifolds," J. Diff. Geom. 48, 91-133 (1998)

[8] R. Bott, A. S. Cattaneo: "Integral invariants of 3-manifolds. II," J. Diff. Geom. $1-13(1999)$

[9] R. Bott, C. Taubes: "On the self-linking of knots," J. Math. Phys. 35, 52475287 (1994)

[10] J-L. Brylinski: Loop Spaces, Characteristic Classes and Geometric Quantization, Progress in Mathematics, 107, Birkhäuser (1993)

[11] A. S. Cattaneo, P. Cotta-Ramusino, J. Fröhlich, M. Martellini: "Topological BF theories in 3 and 4 dimensions," J. Math. Phys. 36, 6137-6160 (1995)

[12] A. S. Cattaneo, P. Cotta-Ramusino, R. Longoni: "Algebraic stuctures in graph homology," in preparation

[13] A. S. Cattaneo, P. Cotta-Ramusino, M. Rinaldi: "Loop and path spaces and four-dimensional $B F$ theories: connections, holonomies and observables," Commun. Math. Phys. 204, 493-524 (1999)

[14] A. S. Cattaneo, P. Cotta-Ramusino, C. Rossi: "Loop observables for BF theories in any dimension and the cohomology of knots," Lett. Math. Phys. 51, 301-316 (2000)

[15] A. S. Cattaneo, J. Fröhlich, B. Pedrini "Topological Field Theory Interpretation of String Topology", to appear in Commun. Math. Phys. arXiv:math.GT/0202176,

[16] A. S. Cattaneo, C. Rossi: "Higher-dimensional BF theories in the BatalinVilkovisky formalism: The BV action and generalized Wilson loops," Commun. Math. Phys. 221, 591-657 (2001), arXiv:math.QA/0010172

[17] M. Chas, D. Sullivan "String Topology," arXiv:math.GT/9911159

[18] K.-T. Chen: "Iterated path integrals," Bull. Amer. Math. Soc. 83, 831-879 (1977)

[19] J. Fröhlich, C. King: "The Chern-Simons theory and knot polynomials," Commun. Math. Phys. 126, 167-199 (1989)

[20] W. Fulton, R. MacPherson: "Compactification of configuration spaces," Ann. Math. 139, 183-225 (1994)

[21] E. Getzler, J. Jones, S. Petrack: "Differential forms on loop spaces and the cyclic bar complex," Topology 30, 339-371 (1991)

[22] T. G. Goodwillie, M. Weiss: "Embeddings from the point of view of immersion theory: Part II," Geometry \& Topology 3, 103-118 (1999)

[23] E. Guadagnini, M. Martellini, M. Mintchev: "Chern-Simons field theory and link invariants," Nucl. Phys. B 330, 575-607 (1989) 
[24] A. C. Hirshfeld, U. Sassenberg: "Explicit formulation of a third order finite knot invariant derived from Chern-Simons theory," J. of Knot Theory and its Ramifications 5, 805-847 (1996)

[25] M. Kontsevich: "Vassiliev's knot invariants," Adv. Sov. Math. 16, 137-150 (1993)

[26] M. Kontsevich: "Feynman diagrams and low-dimensional topology," First European Congress of Mathematics, Paris 1992, Volume II, Progress in Mathematics, 120, Birkhäuser (1994)

[27] M. Kontsevich: "Deformation quantization of Poisson manifolds. I," arXiv:q-alg/9709040

[28] B. Ndombol, J.-C. Thomas: "On the cohomology algebra of free loop spaces," Topology 41, 85-106 (2002).

[29] S. Poirier: "Rationality results for the configuration space integral of knots," arXiv:math.GT/9901028

[30] D. Sinha: "On the topology of spaces of knots," preprint

[31] S. Smale: "The classification of immersions of spheres in Euclidean spaces," Ann. Math. 69, 327-344 (1959)

[32] D. Thurston: Integral Expressions for the Vassiliev Knot Invariants, AB thesis, Harvard University (1995), arXiv : math.QA/9901110

[33] V. Tourtchine: "Sur l'homologie des espaces de noeuds non-compacts," arXiv:math.QA/0010017, "On the homology of the spaces of long knots" arXiv:math.QA/0105140

[34] V. Tourtchine: private communication

[35] V. Vassiliev: "Cohomology of knot spaces," Adv. in Sov. Math.; Theory of Singularities and its Appl. (ed. V. I. Arnold) AMS, Providence, RI, 23-69 (1990)

[36] V. Vassiliev: "Topology of two-connected graphs and homology of spaces of knots," Differential and symplectic topology of knots and curves (S. L. Tabachnikov, ed.) AMS Transl. Ser. 2. Vol. 190. AMS, Providence, RI, 253-286 (1999)

[37] E. Witten: "Quantum field theory and the Jones polynomial," Commun. Math. Phys. 121, 351-399 (1989)

Mathematisches Institut, Universität Zürich-Irchel, Winterthurerstrasse 190

CH-8057 Zürich, Switzerland

Dipartimento di Fisica, Università degli Studi di Milano \& INFN Sezione di Milano

Via Celoria, 16, I-20133 Milano, Italy

Dipartimento di Matematica "G. Castelnuovo", Università di Roma "La Sapienza" Piazzale Aldo Moro, 5, I-00185 Roma, Italy

Email: asc@math.unizh.ch, cotta@mi.infn.it, longoni@mat.uniroma1.it

Received: 2 August 2002

Algebraic 83 Geometric Topology, Volume 2 (2002) 